The University of Southern Mississippi

The Aquila Digital Community

Faculty Publications

4-14-2021

\title{
Engineering Donor-Acceptor Conjugated Polymers for High- Performance and Fast-Response Organic Electrochemical Transistors
}

Hanyu Jia

Peking University

Zhen Huang

College of Chemistry and Molecular Engineering, Peking University, 476330258@qq.com

Peiyun Li

Peking University

Song Zhang

University of Southern Mississippi, song.zhang@usm.edu

Yunfei Wang

University of Southern Mississippi, yunfei.wang@usm.edu

See next page for additional authors

Follow this and additional works at: https://aquila.usm.edu/fac_pubs

Part of the Materials Chemistry Commons

\section{Recommended Citation}

Jia, H., Huang, Z., Li, P., Zhang, S., Wang, Y., Wang, J., Gu, X., Lei, T. (2021). Engineering Donor-Acceptor Conjugated Polymers for High-Performance and Fast-Response Organic Electrochemical Transistors. Journal of Materials Chemistry C, 9(14), 4927-4934.

Available at: https://aquila.usm.edu/fac_pubs/18873

This Article is brought to you for free and open access by The Aquila Digital Community. It has been accepted for inclusion in Faculty Publications by an authorized administrator of The Aquila Digital Community. For more information, please contact Joshua.Cromwell@usm.edu. 


\section{Authors}

Hanyu Jia, Zhen Huang, Peiyun Li, Song Zhang, Yunfei Wang, Jie Yu Wang, Xiaodan Gu, and Ting Lei

This article is available at The Aquila Digital Community: https://aquila.usm.edu/fac_pubs/18873 


\title{
Engineering Donor-Acceptor Conjugated Polymers for High-Performance and
}

\section{Fast-Response Organic Electrochemical Transistors}

\author{
Hanyu Jia ${ }^{1, \ddagger}$, Zhen Huang ${ }^{2, \ddagger}$, Peiyun $\mathrm{Li}^{1, \$}$, Song Zhang ${ }^{3}$, Yunfei Wang ${ }^{3}$, Jie-Yu Wang ${ }^{2}$, Xiaodan Gu ${ }^{3}$, and Ting \\ $\operatorname{Lei}^{1,4, *}$ \\ ${ }^{1}$ Key Laboratory of Polymer Chemistry and Physics of Ministry of Education, Department of Materials Science and Engineering, \\ College of Engineering, Peking University, Beijing 100871, China. \\ ${ }^{2}$ College of Chemistry and Molecular Engineering, Peking University, Beijing 100871, China. \\ ${ }^{3}$ School of Polymer Science and Engineering, The University of Southern Mississippi, Hattiesburg, MS 39406, USA. \\ ${ }^{4}$ Beijing Key Laboratory for Magnetoelectric Materials and Devices, Peking University, Beijing 100871, China. \\ \$These authors contributed equally to this work. \\ *Correspondence and requests for materials should be addressed to T.L. (tinglei@pku.edu.cn).
}

\begin{abstract}
To date, high-performance organic electrochemical transistors (OECTs) are all based on polythiophene systems. Donor-acceptor (D-A) conjugated polymers are expected to be promising materials for OECTs owing to their high mobility and comparatively low crystallinity (good for ion diffusion). However, the OECT performance of D-A polymers lags far behind that of the polythiophenes. Here we synergistically engineered the backbone, side chain of a series of diketopyrrolopyrrole (DPP)-based D-A polymers and found that redox potential, molecular weight, solution processability, and film microstructures are essential to their performance. Among the polymers, $\mathrm{P}(\mathrm{bgDPP}-\mathrm{MeOT} 2)$ exhibited a figure-of-merit $\left(\mu \mathrm{C}^{*}\right)$ of $225 \mathrm{~F} \mathrm{~cm}^{-1} \mathrm{~V}^{-1} \mathrm{~s}^{-1}$, over one order of magnitude higher than previously reported D-A polymers. Besides, the DPP polymers exhibited high hole mobility over $2 \mathrm{~cm}^{2} \mathrm{~V}^{-1} \mathrm{~s}^{-1}$, significantly higher than all D-A polymers employed in OECTs, leading to fast response OECTs with a record low turn-off response time of $30 \mu \mathrm{s}$. The polymer also exhibited better stability than polythiophene systems with current retention of $98.8 \%$ over 700 electrochemical switching cycles. This work provides a systematic solution to unleash the high-performance and fast-response nature of D-A polymers in OECTs.
\end{abstract}

Keywords: conjugated polymers, organic electrochemical transistors, diketopyrrolopyrrole polymers, operation stability, fast response

\section{Introduction}

Organic mixed ionic and electronic conductors (OMIECs), especially polymers, have attracted increasing attention because they can be low-temperature processed, facilely chemically modified, and readily electrochemically doped, while having good ion transport channels and "soft" biological interface. ${ }^{[1]}$ OMIECs have been used for a wide range of applications including sensors, optoelectronics, bioelectronics, and energy 
storage devices. ${ }^{[2]}$ Among these devices, organic electrochemical transistors (OECTs) are particularly attractive because they couple both ionic and electronic inputs to modulate the channel conductance of a transistor in aqueous environment. OECTs have demonstrated their utility in transducing and amplifying low amplitude electrophysiological signals, ${ }^{[3-5]}$ metabolite sensors, ${ }^{[6-8]}$ and neuromorphic computing. ${ }^{[9,10]}$

To evaluate the performance of an OECT material, the following equation is often used (Equation 1):

$$
g_{\mathrm{m}}=\frac{W}{L} \cdot d \cdot \mu \cdot C^{*} \cdot\left(V_{\mathrm{Th}}-V_{\mathrm{GS}}\right)
$$

where $g_{\mathrm{m}}$ is the transconductance in the saturation regime; $I_{\mathrm{DS}}$ is the drain current; $L, W, d$ are the channel length, width, and film thickness, respectively; $\mu$ is the charge carrier mobility; $C^{*}$ is the volumetric capacitance, $V_{\mathrm{Th}}$ is the threshold voltage, and $V_{\mathrm{GS}}$ is the applied gate voltage. Recently, the product of $\mu$ and $C^{*}$ has been proposed to benchmark an OECT material and to realize a better comparison between different materials. $\mu C^{*}$ is the intrinsic property of a material independent of device geometry and bias condition. The higher the $\mu C^{*}$ of the channel material, the more excellent the performance of an OECT under certain device geometry and operating conditions.

Response speed is another important factor of an OECT device, which is particularly important for applications, such as real-time neural signal amplification, high-quality bio-interfacing transmission, and neuromorphic simulation. ${ }^{[4,11,12]}$ Notably, the response speed of OECTs is usually slower than that of organic field-effect transistors (OFETs) because both polymer swelling and ion diffusion are involved. The slow speed substantially limits the applications of OECTs in applications requiring fast signal response. ${ }^{[13]}$ A recent study reveals that when employing an extremely short gate-to-channel length, the response speed of an OECT is limited by hole/electron mobility rather than ion diffusion/redistribution. ${ }^{[14]}$ Therefore, conjugated polymers with high charge carrier mobility are desired for OECTs.

Recently, several thiophene-based conjugated polymers functionalized with ethylene glycol (EG) side chains, e.g. P(g2T-TT) ${ }^{[15]} \mathrm{P}(\mathrm{g} 2 \mathrm{~T}-\mathrm{T}),{ }^{[16]}$ and $\mathrm{P}(\mathrm{g} 2 \mathrm{~T} 2-\mathrm{g} 4 \mathrm{~T} 2)^{[17]}$ have been developed. These polythiophene systems have exhibited high $\mu C^{*}$ over $100 \mathrm{~F} \mathrm{~cm}^{-1} \mathrm{~V}^{-1} \mathrm{~s}^{-1}$, outperforming the conventional materials poly(3,4ethylenedioxythiophene):poly(styrenesulfonate) (PEDOT:PSS) ${ }^{[18]}$ and its derivatives, e.g. Crys-P, ${ }^{[19]}$ in many aspects. However, it is also should be noted that the backbone and the corresponding energy level of polythiophene systems are facing the problem of limited tunability, leaving no room for the development of $\mathrm{n}$ type conjugated polymers, which hampers the application of OECTs in CMOS-like logic circuit and

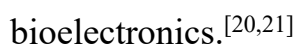

Donor-acceptor (D-A) conjugated polymers have made great advances in the past few years, and their device performances have ranked the top positions in many organic electronics fields, including OFETs, ${ }^{[22]}$ organic photovoltaics (OPVs) ${ }^{[23]}$ and organic thermoelectrics (OTEs). ${ }^{[24]}$ The good backbone planarity, low energetic disorder, and strong interchain interactions make D-A polymers successfully realize high charge carrier mobility with low crystallinity or near amorphous films (Figure 1). ${ }^{[25]}$ Very 
recently, several D-A polymers, using isoindigo (IID), naphthalenediimide (NDI), and pyridine-flanked diketopyrrolopyrrole (PyDPP) building blocks, have been developed as the OECT channel materials. ${ }^{\text {[26- }}$ ${ }^{29]}$ These materials have shown huge potentials for OECTs, including (i) diverse structures that provide vast exploration space and possibilities (ii) large regulation range of frontier orbital energy level to achieve n-type polymers and prevent side-reactions during device operation. ${ }^{[29]}$ Unfortunately, these DA polymers onlyexhibited moderate OECT performance with inferior $\mu C^{*}\left(<10 \mathrm{~F} \mathrm{~cm}^{-1} \mathrm{~V}^{-1} \mathrm{~s}^{-1}\right)$ and slow temporal response ( $>100 \mathrm{~ms}$ ) which have not shown the full potential of D-A polymers from our perspective. The performance-limiting factors of D-A polymers are unknown and essential to alter the situation.

To explore the performance-limiting factors of D-A polymers, here, we report a series of diketopyrrolopyrrole (DPP)-based D-A polymers copolymerized with various donor moieties and grafted with linear or branched EG side chains. DPP was specially chosen because its copolymers have shown high charge carrier mobility in OFETs. ${ }^{[30]}$ Through donor, side chain, polymerization method, and processing solvent engineering, we successfully realized high figure-of-merit OECTs with $\mu C^{*}$ of up to $225 \mathrm{~F} \mathrm{~cm}^{-1} \mathrm{~V}^{-1} \mathrm{~s}^{-1}$, high carrier mobility over $2 \mathrm{~cm}^{2} \mathrm{~V}^{-1} \mathrm{~s}^{-1}$, and fast temporal response. The $\mu C^{*}$ values are over one order of magnitude higher than previously reported D-A copolymers.

\section{Results and discussion}
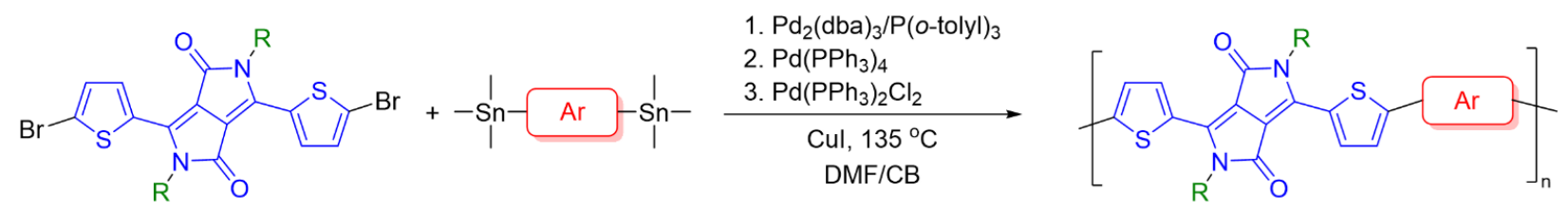

$\mathrm{R}=\mathrm{R}_{1}, \mathrm{R}_{2}$ and $\mathrm{R}_{3}$
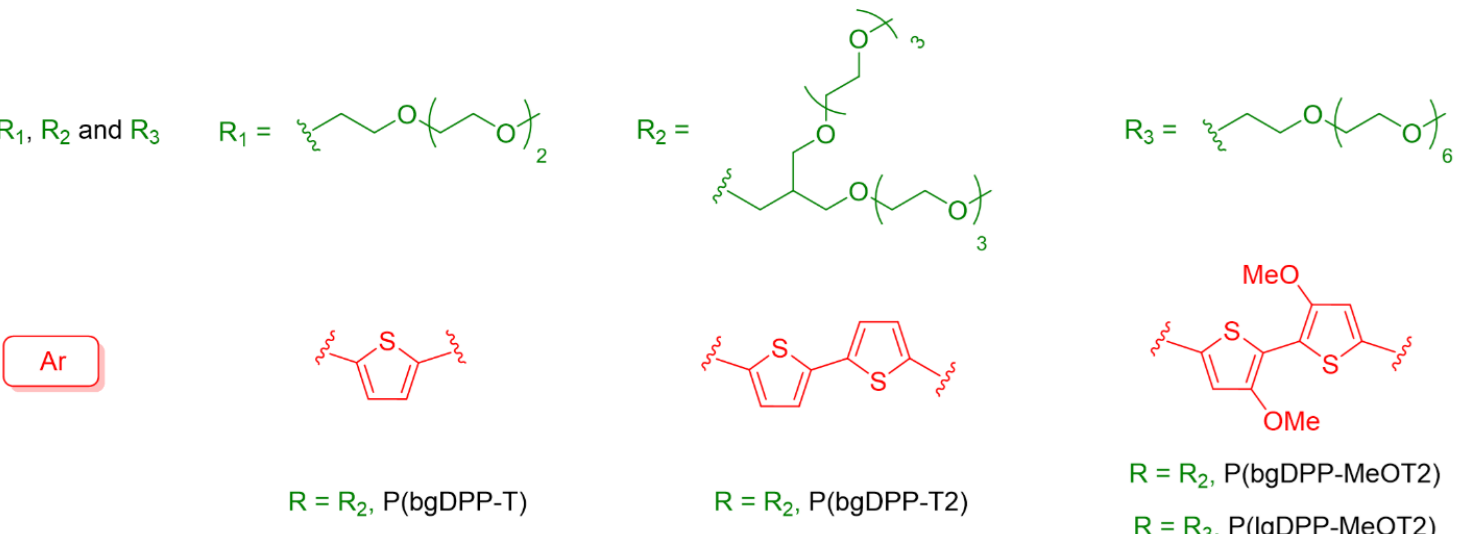

$\mathrm{R}=\mathrm{R}_{2}, \mathrm{P}(\mathrm{bgDPP}-\mathrm{MeOT} 2)$

$\mathrm{R}=\mathrm{R}_{3}, \mathrm{P}(\operatorname{lgDPP}-\mathrm{MeOT} 2)$

Scheme 1 Synthesis and chemical structures of DPP D-A polymers with different donor moieties and grafted with linear or branched ethylene glycol side chains.

Several donor moieties with increased electron-donating properties, e.g. thiophene, 2,2'-bithiophene, and 3,3'methoxy-2,2'-bithiophene (Scheme 1), were used as the donor to tune the highest occupied molecular orbital (HOMO) energy level of the polymers. Similar to previous studies, ${ }^{[15,16]}$ triethylene glycol $\left(\mathrm{R}_{1}\right.$ in Scheme 1$)$ was 
first used as the side chains. However, the strong $\pi-\pi$ stacking interactions of DPP moiety made all the polymers insoluble after polymerization. Therefore, branched EG side chains $\left(R_{2}\right.$ in Scheme 1$)$ were employed to increase the solubility of the polymer. We found that when the monomer grafted with branched EG chains, Stille polymerization using $\mathrm{Pd}_{2}(\mathrm{dba})_{3} / \mathrm{P}(\text { o-tolyl })_{3}$ as the catalyst only yielded oligomers and unreacted monomers. DA polymers grafted with EG chains synthesized with similar polymerization conditions inliterature only showed low molecular weights $(<10 \mathrm{kDa}),{ }^{[26]}$ consistent with our results. After several trials, we found that $\mathrm{Pd}\left(\mathrm{PPh}_{3}\right)_{4}$ or $\mathrm{Pd}\left(\mathrm{PPh}_{3}\right)_{2} \mathrm{Cl}_{2}$ can provide obviously higher molecular weight polymers when using $N, N$-dimethylformamide (DMF) as the solvent. We hypothesize that the branched EG side chains may inhibit the catalytic activity of $\mathrm{Pd}_{2}(\mathrm{dba})_{3} / \mathrm{P}(o \text {-tolyl })_{3}$, probably due to the bulky $\mathrm{P}(o \text {-tolyl })_{3}$. Our observations are supported by previous studies using PEG as the side chains for Stille cross-coupling reactions. ${ }^{[31]}$ To prevent the precipitation of polymers caused by the decreased polymer solubility in DMF, we used DMF/chlorobenzene 1:1 mixture as the solvent. $\mathrm{CuI}$ was added to accelerate the rate of transmetalation for higher molecular weight. ${ }^{[31]}$ We observed that the reaction rate significantly increased as the reaction mixture turned into deep blue in a few minutes, and higher molecular weight polymers can be obtained.

Unlike D-A polymers with alkyl side chains, whose molecular weight can be evaluated using hightemperature GPC (HT GPC, usually $150{ }^{\circ} \mathrm{C}$ ) and 1,2,4-tricholorobenzene (TCB) as the eluent, ${ }^{[24]}$ these polymers did not show reasonable molecular weight or observable signals using HT GPC. This is probably due to the hydrophilic side chains since we observed that even though the polymers are visually dissolved in common aromatic or chlorinated solvents (e.g. $o$-DCB and chloroform), after spin-coating, the polymer films showed large chunks (Figure S1). After trying several eluents, we found that polar solvent hexafluoroisopropanol (HFIP) is a good eluent for molecular weight characterization. When using chloroform as the eluent, the polymers showed high molecular weights with $M_{\mathrm{n}}$ in the range of 61 71 kDa. In contrast, the molecular weights measured using HFIP showed $M_{\mathrm{n}}$ in the range of $26 \sim 30 \mathrm{kDa}$, suggesting the disaggregation of the polymers in HFIP (Table $\mathrm{S} 1$ in the Supporting Information (SI)). These molecular weight values are comparable to their alkyl side chain counterparts. ${ }^{[32]}$ To understand the side-chain effects (linear vs. branched), a longer linear EG side chain $\left(\mathrm{R}_{3}\right.$ in Scheme 1) with the same number of EG segment (- $\left.\mathrm{OCH}_{2} \mathrm{CH}_{2}-\right)$ was used, yielding polymer P(lgDPP-MeOT2). The long linear glycol chains cannot provide enough solubility and only part of the polymers was Soxhlet extracted, giving a low yield of $26 \%$. All the polymers exhibited good thermal stability with the decomposition temperature over $300{ }^{\circ} \mathrm{C}$ (Figure S2).

The optoelectronic properties of the polymers were evaluated using UV-Vis-NIR absorption spectroscopy and cyclic voltammetry (CV). The polymers exhibit a gradual red-shift of absorption maxima when replacing the donor moiety with a stronger electron-donating unit, no matter in solution, film, or annealed film (Figure 1a and Figure S3). DPP polymers containing the most electron-rich donor, namely MeOT2, including P(lgDPPMeOT2), and P(bgDPP-MeOT2), exhibited smaller bandgap than P(bgDPP-T) and P(bgDPP-T2) (Table S2). Therefore, introducing a stronger electron-donating moiety (MeOT2) can remarkably lower the bandgap, largely due to increased HOMO energy levels and enhanced intrachain charge transfer. Interestingly, P(lgDPP-MeOT2) 

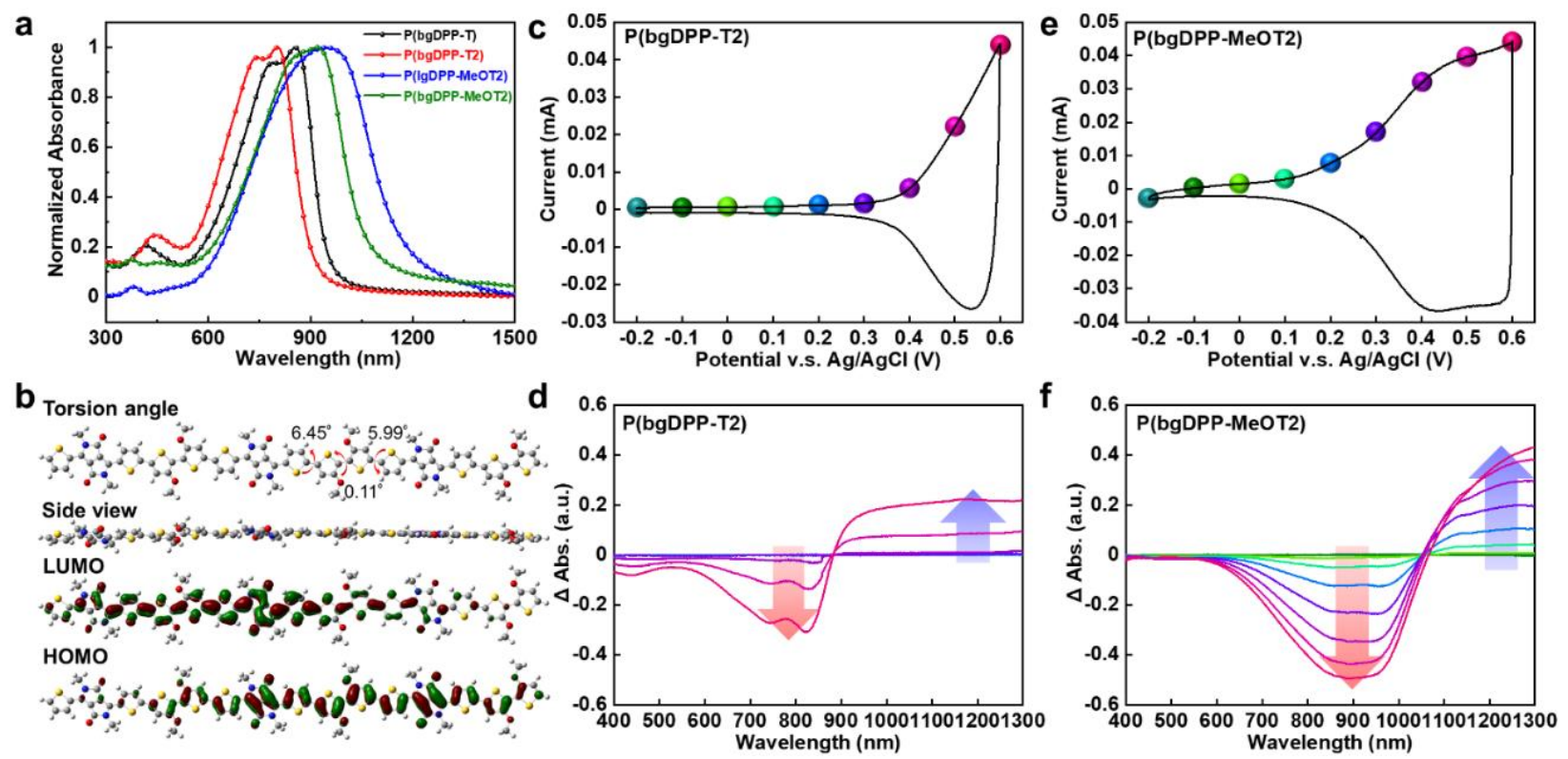

Figure 1 (a) UV-Vis-NIR spectra of spin-coated films of the four polymers after annealing. (b) DFT-optimized geometries and molecular frontier orbitals of the trimer of DPP-MeOT2. Calculations were performed at B3LYP/6-311G(d,p) level. Side chains were replaced with methyl groups to simplify the calculation. (c, e) Cyclic voltammograms and (d, f) differential electrochemical absorption spectra of DPP polymers with branched EG side chains. The color-coding UV-Vis-NIR spectra indicate the applied voltage, ranging from $-0.2 \mathrm{~V}$ to $0.6 \mathrm{~V}$ with an interval of $0.1 \mathrm{~V}$. The variation trends of spectra were highlighted with arrows.

with linear chains exhibited more redshifted absorption than P(bgDPP-MeOT2) with branched side chains. These results were further confirmed by CV measurements (Figure S5-S6 \& Table S2). According to the ionization potentials (IPs) extracted from CV, DPP polymers with MeOT2 donor possess lower IPs of $4.62 \mathrm{eV}$ for $\mathrm{P}($ bgDPP-MeOT2) and $4.35 \mathrm{eV}$ for $\mathrm{P}(\operatorname{lgDPP}-\mathrm{MeOT} 2)$, suggesting that they are more susceptible to oxidation than P(bgDPP-T) and P(bgDPP-T2). DFT calculations showed that all the polymers exhibited planar backbones with small dihedral angles (Figure 1b and Figure S7). The HOMO was delocalized along the backbone, whereas the LUMO was largely localized on the DPP unit. Since linear side chains provide less interchain steric hindrance, we will prove later that $\mathrm{P}(\operatorname{lgDPP}-\mathrm{MeOT} 2)$ has a closer molecular packing. This will lead to more planar backbones and increase the delocalization of the HOMO, thus leading to a higher HOMO level and smaller bandgap.

Spectroelectrochemistry was used to evaluate the electrochemical activity of the DPP polymers, by virtue of its consecutive and controllable electrochemical doping under programmable bias conditions. The changes in absorption spectra and current density upon different potential were monitored in $0.1 \mathrm{M} \mathrm{NaCl}$ aqueous solution. All polymers exhibited reversible and stable electrochemical redox features over $20 \mathrm{CV}$ cycles (Figure S5). Gradually increasing the bias voltage from -0.2 to $0.6 \mathrm{~V}$, three DPP polymers with different donors exhibited different electrochromic activities (Figures 1c-1f \& Figures S7-S8). Concretely, both P(bgDPP-T) and P(bgDPPT2) exhibited a partial extinction of $\pi-\pi^{*}$ absorption band $(650-850 \mathrm{~nm})$ and a gradually increased polaron 
absorption band (1000-1300 nm). It is notably that the absorption variations of P(bgDPP-T) and P(bgDPP-T2) at $750 \mathrm{~nm}$ and $1100 \mathrm{~nm}$ are not obvious until applied bias exceeds $0.3 \mathrm{~V}$, higher than that $(0.1 \mathrm{~V})$ of $\mathrm{P}(\mathrm{bgDPP}-$ MeOT2). To quantify the oxidation degree of the films during the electrochemical scan, differential spectra of DPP polymers were calculated to highlight the absorption variation by subtracting the spectrum of each film recorded under their neutral states (Figure 2). ${ }^{[33]}$ Clearly, P(bgDPP-MeOT2) exhibited a more significant absorption variation in the $\pi-\pi^{*}$ absorption band (750-1050 $\left.\mathrm{nm}\right)$ and the polaron absorption band (1050-1300 $\mathrm{nm})$. These results indicate that $\mathrm{P}(\mathrm{bgDPP}-\mathrm{MeOT} 2)$ is more liable to be $\mathrm{p}$-doped in the aqueousenvironment. Similar results were also found in the linear chain polymer P(lgDPP-MeOT2), which is even more facile to be oxidized due to its increased HOMO energy level (Figure S8).
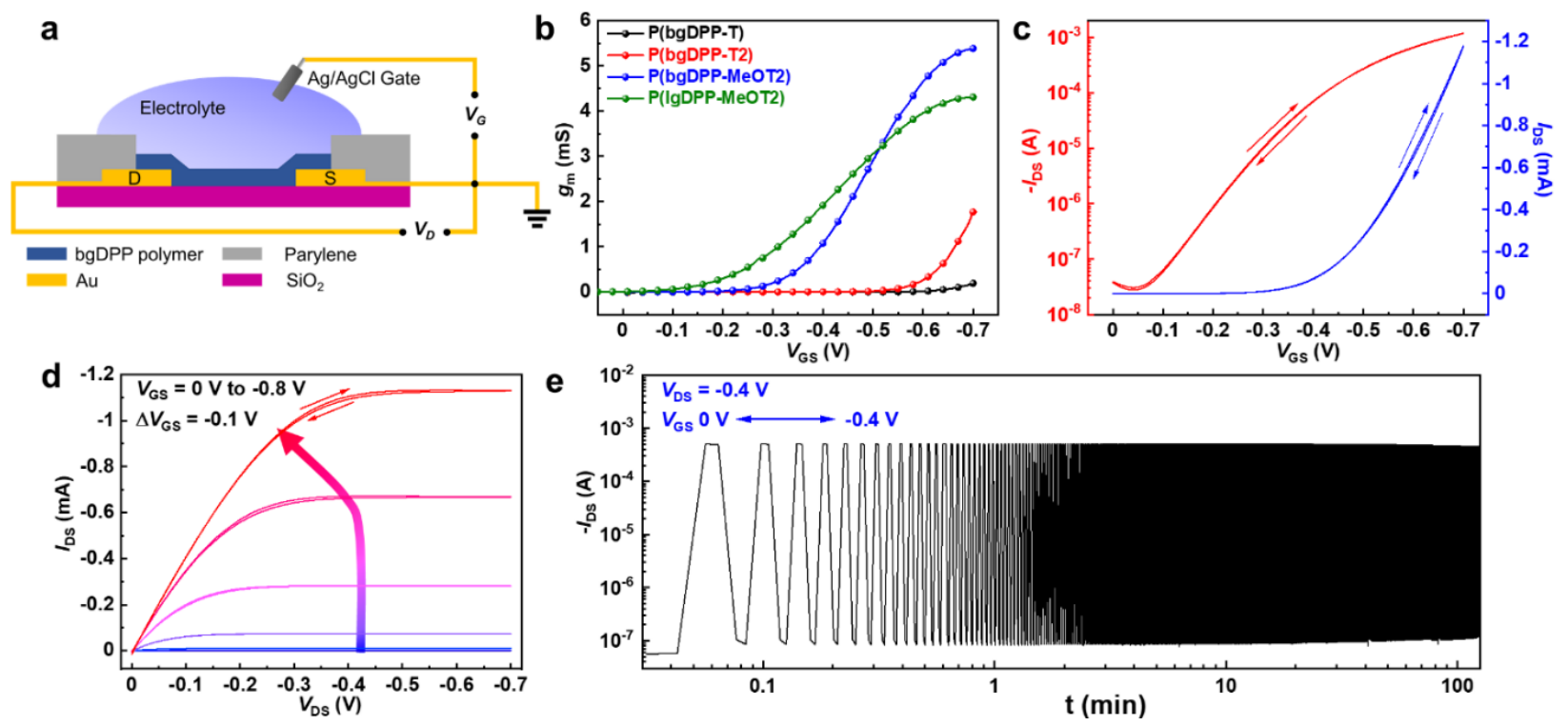

Figure 2 OECT device structure and the device characteristics of $\mathrm{P}(\mathrm{bgDPP}-\mathrm{MeOT} 2)$. (a) Schematic illustration of the OECT device structure in cross-section view and wiring diagram for device operation. (b) Transconductance curves of P(bgDPP-T), P(bgDPPT2), P(lgDPP-MeOT2) and P(bgDPP-MeOT2). (c) Transfer and (d) output characteristics of P(bgDPP-MeOT2) OECTs. VDs = $-0.6 \mathrm{~V}$. (e) Long-term on-off switching of P(bgDPP-MeOT2) operated with the indicated $V_{\mathrm{DS}}, V_{\mathrm{GS}}$ values. Switching on time of $V_{G S}$ and the interval time were set as $2 \mathrm{~s}$ both. All OECTs were measured in $0.1 \mathrm{M} \mathrm{NaCl}$ aqueous solution. W/L $=1000 / 10 \mu \mathrm{m}$ in all devices.

OECTs were fabricated using photolithography and parylene patterning method according to literature. ${ }^{[18,34]}$ We explored many solvents for device fabrication, including $o$-DCB, chlorobenzene (CB), chloroform, trichloroethane, and HFIP. We found that except for HFIP, other solvents cannot provide good device performance ( $g_{\mathrm{m}}$ usually $<0.1 \mathrm{mS}$ for $\mathrm{P}(\mathrm{bgDPP}-\mathrm{MeOT} 2)$ ) using the spin-coating method. When chloroform with the drop-casting method was used, similar performance as HFIP can be obtained but with poor film uniformity. This is probably due to the strong aggregation of the D-A polymers in the solution state (Figure S1). ${ }^{[35]}$ We have noticed that many papers used drop-casting for device fabrication. ${ }^{[16,36]}$ Hence, HFIP and the spin-coating were used for good film uniformity and reproducibility in this work. The figure of merit, $\mu C^{*}$, was extracted for 
performance comparison among different materials. All the DPP polymers exhibited typical p-type OECT behaviors and worked in accumulation mode (Figure 2 and Figure S9). Among all the polymers, P(bgDPPMeOT2) and $\mathrm{P}(\mathrm{lgDPP}-\mathrm{MeOT} 2)$ with the strongest electron-donating moiety MeOT2, exhibited high $g_{\mathrm{m}}$ and high $\mu C^{*}$ values (Table 1). P(bgDPP-MeOT2) exhibited the best OECT performance with a maximum transconductance of up to $5.33 \mathrm{mS}$ with a film thickness of $64 \mathrm{~nm}$, and high $\mu C^{*}$ of up to $225 \mathrm{~F} \mathrm{~cm}^{-1} \mathrm{~V}^{-1} \mathrm{~s}^{-1}$. $\mathrm{P}(\mathrm{bgDPP}-\mathrm{MeOT} 2)$ showed negligible hysteresis during the forward and backward scans, suggesting its good and facile ion transport properties (Figure $2 \mathrm{c} \& 2 \mathrm{~d}$ ). With linear side chains, P(lgDPP-MeOT2) also exhibited outstanding OECT performance with high $\mu C^{*}$ of $174 \pm 25 \mathrm{~F} \mathrm{~cm}^{-1} \mathrm{~V}^{-1} \mathrm{~s}^{-1}$ (Figure S9). In contrast, $\mathrm{P}(\mathrm{bgDPP}-\mathrm{T} 2$ ) and $\mathrm{P}(\mathrm{bgDPP}-\mathrm{T})$ showed inferior OECT performance with $\mu C^{*}$ values of $42 \pm 10$ and $5.9 \pm 0.7 \mathrm{~F} \mathrm{~cm}^{-1} \mathrm{~V}^{-1} \mathrm{~s}^{-1}$. Thus, the electron-donating properties play an important role in the OECT performance of the DPP polymers. Notably, polymer containing MeOT2 moiety showed lower threshold voltage $\left(V_{\mathrm{Th}}\right)$ than that containing $\mathrm{T}$ and $\mathrm{T} 2$ moieties. Interestingly, P(lgDPP-MeOT2) with linear side chains showed even lower $V_{\mathrm{Th}}$ (Figure $\mathrm{S} 11$ ). These results are consistent with the $\mathrm{CV}$ and spectroelectrochemistry studies. The criteria to judge whether a device works in OECT mode or electrolyte-gated organic field effect transistor (EGOFET) mode is the channel thickness dependence. ${ }^{[4,37]}$ OECTs with different film thicknesses were also fabricated (Figure S10). Our devices showed clear film thickness dependent transconductance, suggesting that they indeed work in OECT mode. $\mathrm{P}\left(\right.$ bgDPP-MeOT2) and $\mathrm{P}(\operatorname{lgDPP}-\mathrm{MeOT} 2)$ show much higher $\mu C^{*}$ values than other D-A copolymer OECT materials, e.g. $5.4 \mathrm{~F} \mathrm{~cm}^{-1} \mathrm{~V}^{-1} \mathrm{~s}^{-1}$ for PIBET-AO, $0.18 \mathrm{~F} \mathrm{~cm}^{-1} \mathrm{~V}^{-1} \mathrm{~s}^{-1}$ for $\mathrm{P}(\mathrm{gNDI}-\mathrm{g} 2 \mathrm{~T}$ ) (Figure 3f, Figure $\mathrm{S} 12$ \& Table S3). ${ }^{[26,27]}$ The performance of the DPP polymers also outperforms most of the polythiophene systems that have been developed for many years.

Table 1 Summary of the OECTs Performance and Molecular Packing for the DPP Polymers. ${ }^{\text {a) }}$

\begin{tabular}{|c|c|c|c|c|c|c|c|c|c|c|c|}
\hline Polymer & $d[\mathrm{~nm}]^{\mathrm{a})}$ & $\begin{array}{l}g_{\mathrm{m}, \max } \\
{[\mathrm{mS}]^{\mathrm{a})}}\end{array}$ & $I_{\text {on/off }}$ & $\begin{array}{c}V_{\mathrm{Th}} \\
{[\mathrm{V}]^{\mathrm{b})}}\end{array}$ & $\begin{array}{c}\mu \\
{\left[\mathrm{cm}^{2} \mathrm{~V}^{-1} \mathrm{~s}^{-1}\right]^{\mathrm{c})}}\end{array}$ & $\begin{array}{c}C^{*} \\
{\left[\mathrm{~F} \mathrm{~cm}^{-3}\right]}\end{array}$ & $\begin{array}{c}\mu C^{*} \\
{\left[\mathrm{~F} \mathrm{~cm}^{-2} \mathrm{~V}^{-1} \mathrm{~s}^{-1}\right]^{\mathrm{d})}}\end{array}$ & $\begin{array}{c}\tau_{\mathrm{on}} \\
{[\mu \mathrm{s}]}\end{array}$ & $\begin{array}{l}\tau_{\text {off }} \\
{[\mu \mathrm{s}]}\end{array}$ & $\begin{array}{c}d_{\text {lamellar }} \\
{[\AA ̊]}\end{array}$ & $\begin{array}{l}d_{\pi \cdot \pi} \\
{[\AA]}\end{array}$ \\
\hline $\mathrm{P}($ bgDPP-T) & $29.1 \pm 0.8$ & 0.019 & $2.2 \times 10^{3}$ & -0.60 & $1.59 \pm 0.15$ & $3.7 \pm 0.1$ & $5.9 \pm 0.7$ & - & - & 22.7 & 3.57 \\
\hline P(bgDPP-T2) & $72.5 \pm 0.9$ & 0.403 & $1.8 \times 10^{5}$ & -0.57 & $0.50 \pm 0.11$ & $84.1 \pm 1.5$ & $42 \pm 10$ & - & - & 20.7 & 3.51 \\
\hline P(lgDPP-MeOT2) & $60.9 \pm 0.4$ & 7.04 & $4.9 \times 10^{4}$ & -0.17 & $2.15 \pm 0.27$ & $80.8 \pm 1.4$ & $174 \pm 25$ & 578 & 63 & 18.6 & 3.45 \\
\hline P(bgDPP-MeOT2) & $64.1 \pm 2.4$ & 5.33 & $1.7 \times 10^{5}$ & -0.33 & $1.63 \pm 0.14$ & $120.0 \pm 2.4$ & $195 \pm 21$ & 516 & 30 & 20.7 & 3.55 \\
\hline
\end{tabular}

All the OECT devices were operated in a $0.1 \mathrm{M} \mathrm{NaCl}$ aqueous solution. ${ }^{\text {a) }} 14$ devices with the same channel dimensions were tested and counted for each polymer $(W / L=100 / 10 \mu \mathrm{m}), V_{\mathrm{DS}}=-0.6 \mathrm{~V}$; ${ }^{\text {b) }}$ The threshold voltage, $V_{\mathrm{Th}}$, was determined by extrapolating the corresponding $I_{\mathrm{DS}}{ }^{1 / 2} v S . V_{\mathrm{GS}}$ plots; ${ }^{\mathrm{c})}$ Charge carrier mobility $\mu$ was calculated from the $\mu C^{*}$ and the measured volumetric capacitance $C^{*}$; d) Materials' figure of merit $\mu C^{*}$ were calculated from the measured transconductance.

Stressing measurements upon continuous biasing and long-term on-off switching tests were performed to demonstrate the stable operation of P(bgDPP-MeOT2) statically and dynamically. The drain current of the $\mathrm{P}(\mathrm{bgDPP}-\mathrm{MeOT}$ ) devices stayed almost unchanged atlow and moderate DC bias voltages, after continuous stressing for 10 minutes, while higher biasing condition only leads to a slight loss of $\sim 1.7 \%$ on drain current ( $V_{\mathrm{DS}}$ 
$\left.=V_{\mathrm{GS}}=-0.6 \mathrm{~V}\right)$ (Figure S13). Moreover, long-term on-off switching cycle tests of $\mathrm{P}(\mathrm{bgDPP}-\mathrm{MeOT} 2)$ were also monitored (Figure 2e). The P(bgDPP-MeOT2) device exhibited good stability with current retention of $98.8 \%$ for 700 switching cycles and $89 \%$ for over 3000 cycles (Figure 2e \& Figure S14), better than current state-ofthe-art polythiophene based OECT channel materials. ${ }^{[15,17]}$ Hence, $\mathrm{P}(\mathrm{bgDPP}-\mathrm{MeOT} 2)$ also possesses outstanding stability upon continuous operation.
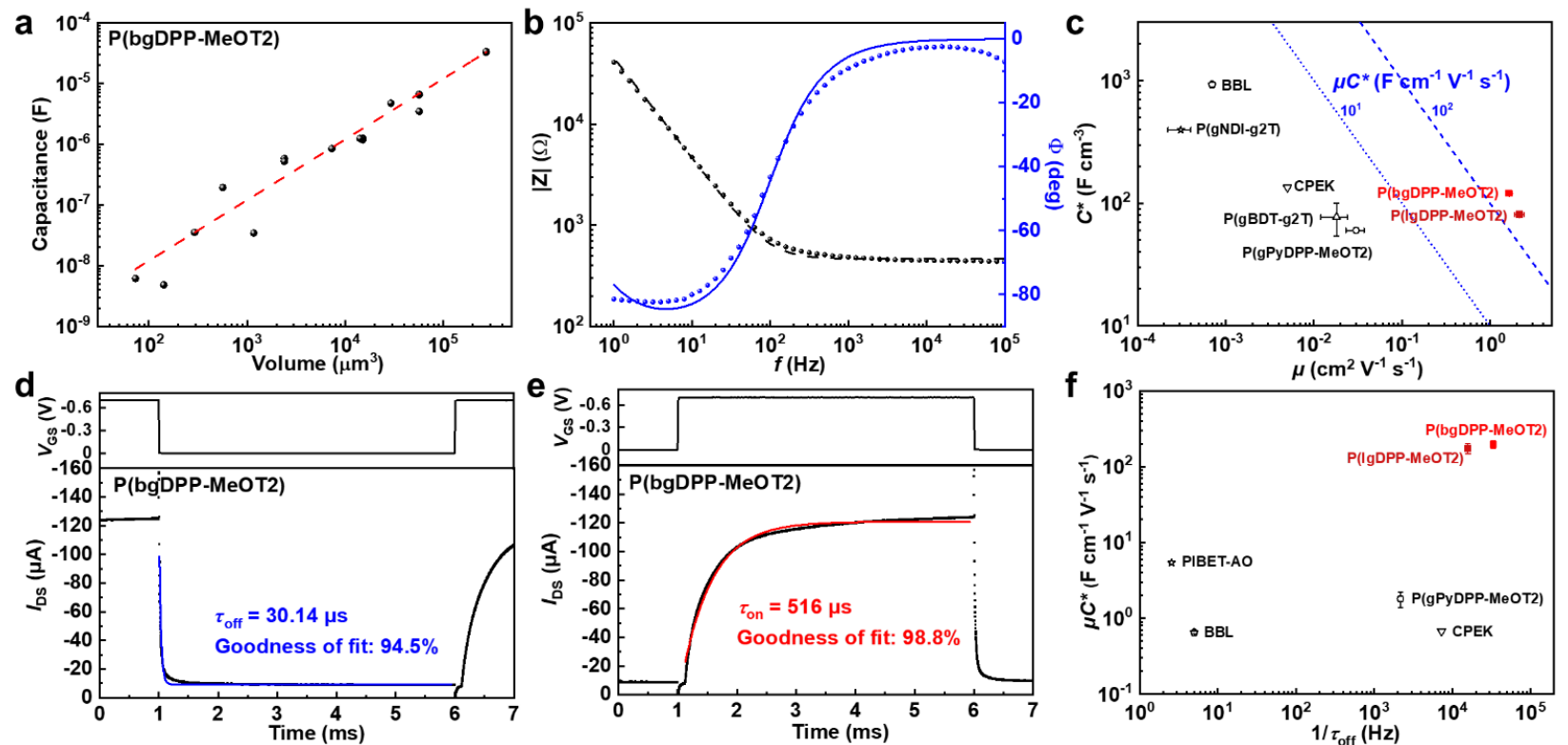

Figure 3 Capacitive, and transient behaviours of P(bgDPP-MeOT2). (a) Volume-capacitance relationship of P(bgDPP-MeOT2) was measured through the electrochemical impedance spectrum. The linear fit to the capacitance data was marked with the red dashed line. (b) The corresponding Bode and phase plot of P(bgDPP-MeOT2) with a channel area of $1 \mathrm{~mm}^{2}$ and thickness of $56.8 \pm 4.2 \mathrm{~nm}$. Data fits were performed via the equivalent circuit of $R_{\mathrm{S}}\left(R_{\mathrm{p}} \| C\right)$. (c) Performance comparison via $2 \mathrm{D} \mu-C^{*}$ plot for P(lgDPP-MeOT2), P(bgDPP-MeOT2), and other reported D-A polymer materials for OECTs. (d, e) Off- \& on-time constant of $\mathrm{P}(\mathrm{bgDPP}-\mathrm{MeOT}$ ) obtained by applying a gate voltage pulse with a time scale of $5 \mathrm{~ms}$. Blue and red lines were fitted through exponential decay function. $W / L=100 / 10 \mu \mathrm{m}$ and $d=34.8 \pm 0.8 \mathrm{~nm}$. (f) Performance comparison via $2 \mathrm{D} \mu C^{*}-1 / \tau_{\text {off }} \mathrm{plot}$ for P(lgDPPMeOT2), P(bgDPP-MeOT2), and other reported D-A polymer materials for OECTs.

To further understand the volumetric doping process of DPP polymers, the electrochemical impedance spectroscopy (EIS) technique was used. Gold electrodes coated with polymer films with certain areas and thicknesses were served as the working electrode with respect to $\mathrm{Pt}$ mesh as the counter electrode and $\mathrm{Ag} / \mathrm{AgCl}$ pellet as the reference electrode. The effective capacitance could be extracted by fitting their EIS data via an equivalent circuit model $\left(R_{\mathrm{s}}\left(R_{\mathrm{p}} \| C\right)\right)$, i.e. a capacitor $(C)$ connects a resistor $\left(R_{\mathrm{p}}\right)$ in parallel and further with a resistor $\left(R_{\mathrm{s}}\right)$ in series (Figure $\left.3 \mathrm{~b}\right)$. The extracted capacitances of $\mathrm{P}(\mathrm{bgDPP}-\mathrm{MeOT} 2)$ upon different channel volumes were plotted, exhibiting a good linear relationship with the channel volume (Figure 3a). The volumetric capacitance $\left(C^{*}\right)$ was extracted with a value of $120.0 \pm 2.4 \mathrm{~F} \mathrm{~cm}^{-3}$. With linear EG chains, P(lgDPP-MeOT2) showed a volumetric capacitance of $80.8 \pm 1.4 \mathrm{~F} \mathrm{~cm}^{-3}$ (Figure S15 \& Table 1), lower than that of P(bgDPP- 
MeOT2). Based on the $\mu C^{*}$ and $C^{*}$ values, the hole mobility $(\mu)$ of both MeOT2 polymers can be calculated. $\mathrm{P}(\mathrm{bgDPP}-\mathrm{MeOT} 2)$ showed hole mobility of $1.63 \pm 0.14 \mathrm{~cm}^{2} \mathrm{~V}^{-1} \mathrm{~s}^{-1}$, and P(lgDPP-MeOT2) showed higher hole mobility of $2.15 \pm 0.27 \mathrm{~cm}^{2} \mathrm{~V}^{-1} \mathrm{~s}^{-1}$ (Table 1). The mobility values are very close to their alkyl side chain counterparts measured in OFETs. ${ }^{[32,38]}$ In OFETs, after introducing linear side chains, the mobility will also increase, largely due to less steric hindrance at the branching positions and a closer $\pi-\pi$ stacking distance. ${ }^{[39,40]}$

To evaluate the response speed of $\mathrm{P}(\mathrm{bgDPP}-\mathrm{MeOT} 2)$, time constants during turn-on and turn-off operation were both measured. As depicted in Figure $3 \mathrm{~d} \& 3 \mathrm{e}$, after applying a $5 \mathrm{~ms}$ pulse voltage on the $\mathrm{Ag} / \mathrm{AgCl}$ gate, temporal responses of drain current were recorded and fitted with the exponential decay function as described by the equation below, ${ }^{[41]}$

$$
I_{\mathrm{DS}}(t)=I_{\mathrm{DS}, 0}+a \times \exp (-t / \tau)
$$

where $I_{\mathrm{DS}}(\mathrm{t})$ represents the drain current at time $t$ after applying the pulse gate bias, $I_{\mathrm{DS}, 0}$ represents the initial drain current before applying the pulse bias, $a$ is a constant and $\tau$ is the time constant. The off-time constant $\left(\tau_{\text {off }}\right)$ and on-time constant $\left(\tau_{\text {on }}\right)$ were estimated to be $30 \mu$ s and $516 \mu$ s for P(bgDPP-MeOT2, with a channel geometry of $100 \mu \mathrm{m} / 10 \mu \mathrm{m}(W / L)$. Obviously, both off- and on-time constants of P(bgDPP-MeOT2) reach the topperforming level among reported polymers, including D-A polymers and polythiophenes (Figure $3 \mathrm{f} \&$ Table S3). According to literature, the time constant of p-type OECT is mainly dominated by the ion injection process and the removal of holes from the source electrode. Gaining higher hole mobility or volumetric capacitance can effectively enhance the response speed. In specific, $\mathrm{P}(\operatorname{lgDPP}-\mathrm{MeOT} 2)$ also exhibited fast response characteristic on the transient behaviors. On- \& off-time constant of P(lgDPP-MeOT2) under similar channel geometry achieved $578 \mu \mathrm{s}$ and $63 \mu \mathrm{s}$, respectively (Figure S16). To fully compare thecomprehensive performance of $\mathrm{P}(\mathrm{bgDPP}-\mathrm{MeOT} 2)$ with other reported polymers, the reciprocal of on- and off-time constant and the $\mu C^{*}$ are plotted in Figure 3f. ${ }^{[15,16,26,34,42,43]}$
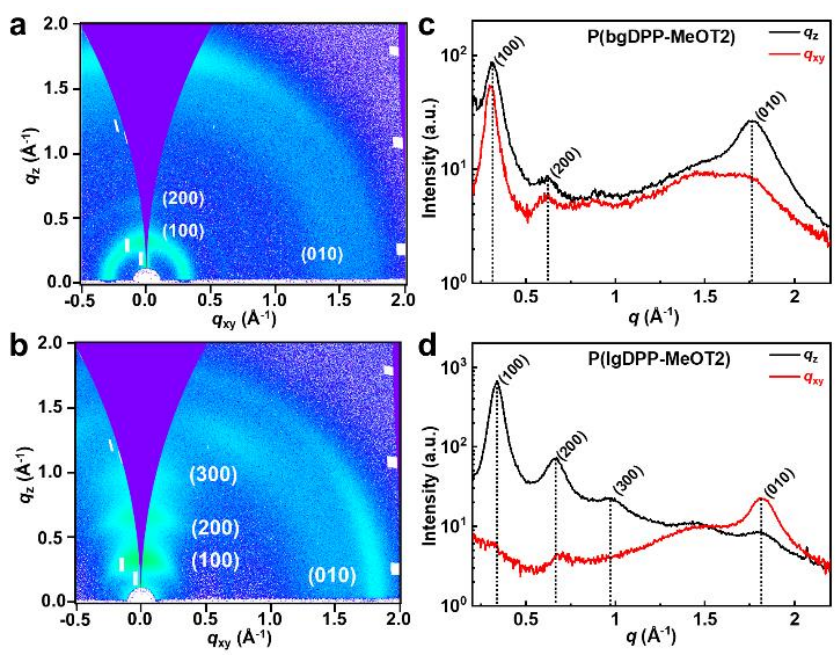

Figure 4 2D-GIWAXS patterns of (a) P(bgDPP-MeOT2) and (b) P(lgDPP-MeOT2); (c-d) The corresponding line cuts of P(bgDPPMeOT2) and P(lgDPP-MeOT2). Cuts along the $q_{\mathrm{xy}}$ direction (red) represent scattering in the plane of the substrate, while the scattering in the $q_{\mathrm{z}}$ direction (black) results from out-of-plane scattering. 
Crystallinity and molecular packing of conjugated polymers strongly influence water uptake, ion transport, and charge carrier transport in the polymer bulk. Two-dimensional (2D) GIWAXS was employed to reveal the differences among the polymers. All bgDPP polymers oriented preferably in a face-on fashion, while $\mathrm{P}(\operatorname{lgDPP}$ MeOT2) with linear EG chains, oriented predominantly with edge-on (Figure 4). P(lgDPP-MeOT2) exhibited a closer $\pi-\pi$ stacking distance of $3.45 \AA$, smaller than those of the bgDPP polymers (3.51-3.57 $\AA$ ), consisting with our previous absorption spectra analysis and mobility results (Figure S17). In addition, P(lgDPP-MeOT2) also exhibited three orders of lamellar scattering peaks, (100), (200), and (300), indicating the well-packed polymer side chains compared with those with branched side chains. For conjugated polymers with highly ordered crystallites, the injection of hydrated ions into polymer bulk may induce destruction of morphology and then impede charge transport between adjacent crystallites. ${ }^{[13,44]}$ Therefore, less ordered packing of P(bgDPP-MeOT2) might contribute to the enhanced penetration of hydrated ions into the polymer bulk (higher $C^{*}$ ) and faster temporal response, though its hole mobility is slightly sacrificed.

\section{Conclusions}

In conclusion, we have systematically explored the influences of the donor, side chain, molecular weight, and processing conditions to solve the low-performance issue in D-A conjugated polymers. The high-performance of $\mathrm{P}(\mathrm{bgDPP}-\mathrm{MeOT} 2)$ can be attributed to the following molecular design and device fabrication considerations: (i) strong electron-donating moiety MeOT2 reduces the ionization potential of DPP polymers, leading to a low threshold voltage and high volumetric capacitance; (ii) the branched EG chains guarantee enough solubility for high molecular weight polymers and also facilitate ion injection/ejection in the polymer bulk; (iii) optimized polymerization method allows comparable molecular weight and hole mobility as its alkyl side chain counterpart; (iv) the "uncommon" polar solvent HFIP is used to disaggregate the polymers for better film quality. These efforts lead to a high $\mu C^{*}\left(>200 \mathrm{~F} \mathrm{~cm}^{-1} \mathrm{~V}^{-1} \mathrm{~s}^{-1}\right)$, high hole mobility $\left(>2 \mathrm{~cm}^{2} \mathrm{~V}^{-1} \mathrm{~s}^{-1}\right)$, and fast response $\left(\tau_{\text {off }} 30\right.$ $\mu \mathrm{s} ; \tau_{\text {on }} 516 \mu \mathrm{s}$ ), much higher than other D-A polymer-based OECT materials (Table S3). Considering that most n-type conjugated polymers are based on D-A copolymers, we believe that our study will not only benefit highperformance and fast-response p-type OECT materials but also will be valuable for $\mathrm{n}$-type OECT materials whose performance lags far behind that of the p-type ones.

\section{Conflicts of interest}

There are no conflicts to declare.

\section{Acknowledgements}

This work is supported by the Guangdong Science and Technology Project (2019B010934001) and the Beijing Natural Science Foundation (2192020). The computational part is supported by Highperformance Computing Platform of Peking University. The scattering component in this manuscript is supported by U.S. Department of Energy, Office of Science, Office of Basic Energy Science under award number of DE-SC0019361.

Hanyu Jia, Zhen Huang, and Peiyun Li contributed equally to this work. 


\section{References}

[1] J. Rivnay, R. M. Owens, G. G. Malliaras, Chem. Mater., 2013, 26, 679.

[2] B. D. Paulsen, K. Tybrandt, E. Stavrinidou, J. Rivnay, Nat. Mater., 2020, 19, 13.

[3] G. D. Spyropoulos, J. N. Gelinas, D. Khodagholy, Sci. Adv., 2019, 5, eaau7378.

[4] J. Rivnay, P. Leleux, M. Ferro, M. Sessolo, A. Williamson, D. A. Koutsouras, D. Khodagholy, M. Ramuz, X. Strakosas,

R. M. Owens, C. Benar, J. M. Badier, C. Bernard, G. G. Malliaras, Sci. Adv., 2015, 1, e1400251.

[5] V. Venkatraman, J. T. Friedlein, A. Giovannitti, I. P. Maria, I. McCulloch, R. R. McLeod, J. Rivnay, Adv. Mater., 2018, 5, 1800453.

[6] O. Parlak, S. T. Keene, A. Marais, V. F. Curto, A. Salleo, Sci. Adv., 2018, 4, eaar2904.

[7] A. M. Pappa, D. Ohayon, A. Giovannitti, I. P. Maria, A. Savva, I. Uguz, J. Rivnay, I. McCulloch, R. M. Owens, S. Inal, Sci. Adv., 2018, 4, eaat0911.

[8] P. Li, T. Lei, L. Ding, Sci. Bull., 2020, 65, 1141.

[9] Y. van de Burgt, E. Lubberman, E. J. Fuller, S. T. Keene, G. C. Faria, S. Agarwal, M. J. Marinella, A. Alec Talin, A. Salleo, Nat. Mater., 2017, 16, 414.

[10] Y. van de Burgt, A. Melianas, S. T. Keene, G. Malliaras, A. Salleo, Nat. Electron., 2018, 1, 386.

[11] D. Khodagholy, T. Doublet, P. Quilichini, M. Gurfinkel, P. Leleux, A. Ghestem, E. Ismailova, T. Herve, S. Sanaur, C. Bernard, G. G. Malliaras, Nat. Commun., 2013, 4, 1575.

[12] P. Gkoupidenis, N. Schaefer, B. Garlan, G. G. Malliaras, Adv. Mater., 2015, 27, 7176.

[13] R. Giridharagopal, L. Q. Flagg, J. S. Harrison, M. E. Ziffer, J. Onorato, C. K. Luscombe, D. S. Ginger, Nat. Mater., 2017, 16, 737.

[14] C. Cea, G. D. Spyropoulos, P. Jastrzebska-Perfect, J. J. Ferrero, J. N. Gelinas, D. Khodagholy, Nat. Mater., 2020, $19,679$.

[15] A. Giovannitti, D. T. Sbircea, S. Inal, C. B. Nielsen, E. Bandiello, D. A. Hanifi, M. Sessolo, G. G. Malliaras, I. McCulloch, J. Rivnay, Proc. Natl. Acad. Sci. U. S. A., 2016, 113, 12017.

[16] C. B. Nielsen, A. Giovannitti, D. T. Sbircea, E. Bandiello, M. R. Niazi, D. A. Hanifi, M. Sessolo, A. Amassian, G. G. Malliaras, J. Rivnay, I. McCulloch, J. Am. Chem. Soc., 2016, 138, 10252.

[17] M. Moser, T. C. Hidalgo, J. Surgailis, J. Gladisch, S. Ghosh, R. Sheelamanthula, Q. Thiburce, A. Giovannitti, A. Salleo, N. Gasparini, A. Wadsworth, I. Zozoulenko, M. Berggren, E. Stavrinidou, S. Inal, I. McCulloch, Adv. Mater., 2020, 32, e2002748.

[18] S. Inal, G. G. Malliaras, J. Rivnay, Nat. Commun., 2017, 8, 1767.

[19] S. M. Kim, C. H. Kim, Y. Kim, N. Kim, W. J. Lee, E. H. Lee, D. Kim, S. Park, K. Lee, J. Rivnay, M. H. Yoon, Nat. Commun., 2018, 9, 3858.

[20] H. Sun, M. Vagin, S. Wang, X. Crispin, R. Forchheimer, M. Berggren, S. Fabiano, Adv. Mater., 2018, 30, 1704916.

[21] M. Kawan, T. C. Hidalgo, W. Du, A.-M. Pappa, R. M. Owens, I. McCulloch, S. Inal, Mater. Horiz., $2020,7,2348$.

[22] J. Yang, Z. Y. Zhao, S. Wang, Y. L. Guo, Y. Q. Liu, Chem, 2018, 4, 2748.

[23] H. Jia, T. Lei, J. Mater. Chem. C, 2019, 7, 12809.

[24] X. Yan, M. Xiong, J. T. Li, S. Zhang, Z. Ahmad, Y. Lu, Z. Y. Wang, Z. F. Yao, J. Y. Wang, X. Gu, T. Lei, J. Am. Chem. Soc., 2019, 141, 20215.

[25] S. Fratini, M. Nikolka, A. Salleo, G. Schweicher, H. Sirringhaus, Nat. Mater., 2020, 19, 491.

[26] Y. Wang, E. Zeglio, H. Liao, J. Xu, F. Liu, Z. Li, I. P. Maria, D. Mawad, A. Herland, I. McCulloch, W. Yue, Chem. Mater., 2019, 31, 9797. 
[27] A. Giovannitti, C. B. Nielsen, D. T. Sbircea, S. Inal, M. Donahue, M. R. Niazi, D. A. Hanifi, A. Amassian, G. G. Malliaras, J. Rivnay, I. McCulloch, Nat. Commun., 2016, 7, 13066.

[28] A. Giovannitti, I. P. Maria, D. Hanifi, M. J. Donahue, D. Bryant, K. J. Barth, B. E. Makdah, A. Savva, D. Moia, M. Zetek, P. R. F. Barnes, O. G. Reid, S. Inal, G. Rumbles, G. G. Malliaras, J. Nelson, J. Rivnay, I. McCulloch, Chem. Mater., 2018, 30, 2945.

[29] A. Giovannitti, R. B. Rashid, Q. Thiburce, B. D. Paulsen, C. Cendra, K. Thorley, D. Moia, J. T. Mefford, D. Hanifi, D. Weiyuan, M. Moser, A. Salleo, J. Nelson, I. McCulloch, J. Rivnay, Adv. Mater., 2020, 32, e1908047.

[30] Z. Yi, S. Wang, Y. Liu, Adv. Mater., 2015, 27, 3589.

[31] B. Carsten, F. He, H. J. Son, T. Xu, L. Yu, Chem. Rev., 2011, 111, 1493.

[32] R. Di Pietro, T. Erdmann, J. H. Carpenter, N. Wang, R. R. Shivhare, P. Formanek, C. Heintze, B. Voit, D. Neher, H. Ade, A. Kiriy, Chem. Mater., 2017, 29, 10220.

[33] P. Schmode, D. Ohayon, P. M. Reichstein, A. Savva, S. Inal, M. Thelakkat, Chem. Mater., 2019, $31,5286$.

[34] D. Khodagholy, J. Rivnay, M. Sessolo, M. Gurfinkel, P. Leleux, L. H. Jimison, E. Stavrinidou, T. Herve, S. Sanaur, R. M. Owens, G. G. Malliaras, Nat. Commun., 2013, 4, 2133.

[35] M. Li, H. Bin, X. Jiao, M. M. Wienk, H. Yan, R. A. J. Janssen, Angew. Chem., Int. Ed., 2020, $59,846$.

[36] A. Giovannitti, K. J. Thorley, C. B. Nielsen, J. Li, M. J. Donahue, G. G. Malliaras, J. Rivnay, I. McCulloch, Adv. Funct. Mater., 2018, 28, 1706325.

[37] E. Zeglio, O. Inganas, Adv. Mater., 2018, 30, e1800941.

[38] Y. Li, P. Sonar, S. P. Singh, M. S. Soh, M. van Meurs, J. Tan, J. Am. Chem. Soc., 2011, 133, 2198.

[39] T. Lei, J. H. Dou, J. Pei, Adv. Mater., 2012, 24, 6457.

[40] Z. Wang, Z. Liu, L. Ning, M. Xiao, Y. Yi, Z. Cai, A. Sadhanala, G. Zhang, W. Chen, H. Sirringhaus, D. Zhang, Chem. Mater., 2018, 30, 3090.

[41] D. A. Bernards, G. G. Malliaras, Adv. Funct. Mater., 2007, 17, 3538.

[42] A. Savva, R. Hallani, C. Cendra, J. Surgailis, T. C. Hidalgo, S. Wustoni, R. Sheelamanthula, X. Chen, M. Kirkus, A. Giovannitti, A. Salleo, I. McCulloch, S. Inal, Adv. Funct. Mater., 2020, 30, 1907657.

[43] S. Inal, J. Rivnay, P. Leleux, M. Ferro, M. Ramuz, J. C. Brendel, M. M. Schmidt, M. Thelakkat, G. G. Malliaras, Adv. Mater., 2014, 26, 7450.

[44] L. Q. Flagg, C. G. Bischak, J. W. Onorato, R. B. Rashid, C. K. Luscombe, D. S. Ginger, J. Am. Chem. Soc., 2019, 141, 4345. 


\section{Supporting Information}

for

\section{Engineering Donor-Acceptor Conjugated Polymers for High-Performance and Fast-Response Organic Electrochemical Transistors}

Hanyu Jia ${ }^{1, \neq}$, Zhen Huang ${ }^{2, \neq}$, Peiyun $\mathrm{Li}^{1,+}$, Song Zhang ${ }^{3}$, Yunfei Wang ${ }^{3}$, Jie-Yu Wang ${ }^{2}$, Xiaodan $\mathrm{Gu}^{3}$, and Ting Lei1 ${ }^{1,4, *}$

${ }^{1}$ Key Laboratory of Polymer Chemistry and Physics of Ministry of Education, Department of Materials Science and Engineering, College of Engineering, Peking University, Beijing 100871, China.

${ }^{2}$ College of Chemistry and Molecular Engineering, Peking University, Beijing 100871, China.

${ }^{3}$ School of Polymer Science and Engineering, The University of Southern Mississippi, Hattiesburg, MS 39406, USA.

${ }^{4}$ Beijing Key Laboratory for Magnetoelectric Materials and Devices, Peking University, Beijing 100871, China.

${ }^{\ddagger}$ These authors contributed equally to this work.

*Correspondence and requests for materials should be addressed to T.L. (tinglei@pku.edu.cn).

\section{Table of Contents}

1. Experimental Details

2. Table S1-S3 and Figure S1-S17

3. Synthetic Procedure and Characterization

4. ${ }^{1} \mathrm{H}$ and ${ }^{13} \mathrm{C}$ NMR spectra (Figure S18-S29)

5. References 


\section{Experimental Details}

\section{Materials}

All chemical reagents were purchased and used as received unless otherwise indicated. All air and water sensitive reactions were performed under nitrogen atmosphere. Dichloromethane (DCM), tetrahydrofuran (THF), toluene, and $N, N$-dimethylformamide (DMF) were dried by a JC Meyer solvent drying system.

\section{Chemical Structure and Optoelectronic Property Characterization}

${ }^{1} \mathrm{H}$ NMR and ${ }^{13} \mathrm{C}$ NMR spectra were recorded on Bruker ARX-400 (400 MHz), Bruker AVANCE III (500 $\mathrm{MHz})$, and Bruker 600M (600 MHz). All chemical shifts were reported in parts per million (ppm). ${ }^{1} \mathrm{H}$ NMR chemical shifts were referenced to $\mathrm{CDCl}_{3}(7.262 \mathrm{ppm})$ and $\mathrm{CDCl}_{2} \mathrm{CDCl}_{2}(5.984 \mathrm{ppm}),{ }^{13} \mathrm{C}$ NMR chemical shifts were referenced to $\mathrm{CDCl}_{3}$ (77.00 ppm). Mass spectra were recorded on an AB Sciex-5800 MALDITOF mass spectrometer and a Bruker Solarix XR mass spectrometer. Elemental analyses were performed on Vario EL elemental analyzer. Thermal gravity analyses (TGA) were carried out on a TA Instrument Q600 SDT analyzer. Absorption spectra were recorded on PerkinElmer Lambda 750 UV-Vis spectrometer. Cyclic voltammograms were measured through an electrochemical workstation SP-300 (BioLogic Science Instruments). A standard three-electrode setup was established with employing polymer-coated ITO glass slides as the working electrode (WE), a block of platinum mesh as the counter electrode (CE), and an $\mathrm{Ag} / \mathrm{AgCl}$ pellet (Warner Instruments) as the reference electrode (RE), further calibrated against ferrocene $\left(\mathrm{Fc} / \mathrm{Fc}^{+}\right)$. The measurements were carried out in aqueous solution with $0.1 \mathrm{M} \mathrm{NaCl}$ or in acetonitrile with 0.1 M tetrabutylammonium hexafluorophosphate as the supporting electrolyte with a scan rate of $50 \mathrm{mV} / \mathrm{s}$. Ionization potentials and electron affinity were obtained using the equation: $\mathrm{IP}=\left(E_{\mathrm{Ox}}-E_{\mathrm{Fc} / \mathrm{Fc}}{ }^{+}+4.8\right) \mathrm{eV}, \mathrm{EA}$ $=\left(E_{\mathrm{Red}}-E_{\mathrm{Fc} / \mathrm{Fc}^{+}}{ }^{+}+4.8\right) \mathrm{eV}$. The geometries and frontier orbitals of bgDPP-T, bgDPP-T2, lgDPP-MeOT2, and bgDPP-MeOT2 trimers were calculated at the B3LYP/6-311G(d,p) level using Gaussian 16 software package.

\section{Size Exclusion Chromatography Measurement}

Polymer number-average molecular weight $\left(M_{\mathrm{n}}\right)$ and molecular weight distributions $\left(\mathrm{Ð}=M_{\mathrm{w}} / M_{\mathrm{n}}\right)$ were measured by size exclusion chromatography (SEC). Chloroform SEC analyses were performed on a Waters 1515 instrument equipped with a PLMIXED $7.5 \times 50 \mathrm{~mm}$ guard column and two PLMIXED-C $7.5 \times 300$ columns and a differential refractive index detector using chloroform as the eluent at $35^{\circ} \mathrm{C}$ with a flow rate 
of $1 \mathrm{~mL} \mathrm{~min}^{-1}$. The instrument was calibrated with 10 PS standards, and chromatograms were processed with Waters Breeze software. Hexafluoroisopropanol SEC analyses were performed on a Waters 1515 instrument equipped with a PLMIXED $7.5 \times 50 \mathrm{~mm}$ guard column and two PLMIXED-C $7.5 \times 300$ columns and a differential refractive index detector using hexafluoroisopropanol as the eluent at $35^{\circ} \mathrm{C}$ with a flow rate of 1 $\mathrm{mL} \min ^{-1}$. The instrument was calibrated with 10 PMMA standards, and chromatograms were processed with Waters Breeze software.

\section{AFM and GIWAXS characterization}

Atomic Force Microscopy (AFM) measurements were performed with a Cypher atomic force microscope (Asylum Research, Oxford Instruments). The surface morphology was recorded with a scan rate of 2-3 Hz at AC mode. GIWAXS experiment was performed on Xenocs Xuess 2.0 beamline, with an incident X-ray angle of 0.2 degrees and wavelength of 1.54 angstrom. The scattered signal is collected by Pilatus $1 \mathrm{M}$ detector at a sample to detector distance of $150 \mathrm{~mm}$. Data processing is performed in Igor Pro software with Nika and WAXTools packages.

\section{Spectroelectrochemistry}

Spectroelectrochemistry was performed with an ITO-coated glass slide, spun cast with the polymer solution (3*10-3 $\mathrm{M}$ chloroform solution) at the rotating speed of $500 \mathrm{rpm}$ for $45 \mathrm{~s}$ without any additional processing. These polymer-coated ITO slides were employed as the WE and immersed into the cuvette filled with $0.1 \mathrm{M}$ aqueous $\mathrm{NaCl}$ solution, following with the use of Pt mesh (CE) and $\mathrm{Ag} / \mathrm{AgCl}$ pellet (RE). A PerkinElmer Lambda $750 \mathrm{UV}$-vis spectrometer was used with the beam path passing through the electrolyte-filled cuvette and polymer-coated ITO samples. A background spectrum with cuvette/electrolyte/ITO was recorded before a potential was applied to the cell. The potential was applied to the WE for $5 \mathrm{~s}$ before the spectra were recorded and lasted for a certain amount of time until the completion of spectrum scanning.

\section{OECT Fabrication and Characterization}

The OECTs fabrication included the deposition and patterning of the metallic electrodes, parylene layer, and polymer in the channel. In detail, the silica substrates were thoroughly cleaned by sonication in acetone, DI water, and isopropyl alcohol, followed by nitrogen blow drying and brief oxygen plasma cleaning. Metal 
pads, interconnects, and source/drain contacts (defining the channel length and width) were patterned by a lift-off process. $5 \mathrm{~nm}$ of chromium and $50 \mathrm{~nm}$ of gold were subsequently deposited using a metal evaporator, and metal lift-off was carried out in acetone. Metal interconnects and pads were insulated by depositing $2 \mu \mathrm{m}$ of parylene-C using a PDS 2010 Labcoater-2, with a 3-(trimethoxysilyl)propyl methacrylate (A-174 Silane) adhesion promoter. A $2 \%$ aqueous solution of industrial cleaner (Micro-90) was subsequently spun coated to act as an anti-adhesive for a second, sacrificial $2 \mu \mathrm{m}$ parylene-C film, which was used to simultaneously define the active channel area, and to pattern the underlying parylene layer. Samples were subsequently patterned with a $5 \mu \mathrm{m}$ thick layer of AZ9260 photoresist and AZ-400K developer. The patterned areas were opened by reactive ion etching with $\mathrm{O}_{2}$ plasma using an LCCP-6A reactive ion etcher (Leuven Instruments). For the polymer film formation in the opened channels, the polymer solution was spun cast on the etched devices with different rotating speeds depending on the desired film thickness. After a peeling-off process of the second sacrificial parylene layer, the OECTs were ready for measurement. The device characterization was performed on the Keithley $4200 \mathrm{SCS}$. $\mathrm{Ag} / \mathrm{AgCl}$ pellet (Warner Instruments) was employed as the gate and immersed into a $0.1 \mathrm{M} \mathrm{NaCl}$ solution, which covers the polymer film in the channel.

\section{Electrochemical Impedance Spectra}

Electrochemical impedance spectra (EIS) were performed on the polymer-coated electrodes using the electrochemical workstation SP-300 (BioLogic Science Instruments). Polymer coated electrodes were patterned as squares with different areas through lithography. These polymer-coated electrodes with glass substrate were employed as the working electrode and fully covered with a $0.1 \mathrm{M} \mathrm{NaCl}$ solution, followed with the employment of Pt mesh (CE) and $\mathrm{Ag} / \mathrm{AgCl}$ pellet (RE) to establish a standard three electrodes system. The capacitances of polymers on electrodes with various sizes were obtained through the potentio-EIS method, with setting the DC offset voltage as the maximum achievable doping for each polymer. The AC amplitude of voltage in the form of sine-wave on the WE was set as $10 \mathrm{mV}$ (RMS) and the frequency was scanned from $100 \mathrm{kHz}$ to $1 \mathrm{~Hz}$. The as-obtained Bode plots or Nyquist plots were fitted to an equivalent circuit, namely the Randle's circuit $R_{\mathrm{s}}\left(R_{\mathrm{p}} \| C\right)$, via the software EC-Lab view. The thickness of the films was determined in the dry state with a DEKTAK profilometer (Bruker). 


\section{Table S1-S3 and Figure S1-S17}

Table S1. Summary of Synthetic Conditions and Molecular Weights of the DPP Polymers.

\begin{tabular}{cccccccc}
\hline Polymer & Catalyst/ligand & Cocatalyst & $\begin{array}{c}\text { Yield } \\
{[\%]}\end{array}$ & $\begin{array}{c}M_{\mathrm{n}}^{\mathrm{a}} \\
{[\mathrm{kDa}]}\end{array}$ & $\mathrm{PDI}^{\mathrm{a}}$ & $\begin{array}{c}M_{\mathrm{n}}^{\mathrm{b}} \\
{[\mathrm{kDa}]}\end{array}$ & $\mathrm{PDI}^{\mathrm{b}}$ \\
\hline P(lg3DPP-T) & $\mathrm{Pd}_{2}(\mathrm{dba})_{3} / \mathrm{P}(\mathrm{o}-\mathrm{tolyl})_{3}$ & $/$ & $20^{\mathrm{c}}$ & $/$ & $/$ & $/$ & $/$ \\
P(bgDPP-T) & $\left.\mathrm{Pd}_{(\mathrm{PPh}}\right)_{4}$ & $\mathrm{CuI}$ & 62 & 69.0 & 2.32 & 29.5 & 2.50 \\
P(bgDPP-T2) & $\left.\mathrm{Pd}_{(\mathrm{PPh}}\right)_{2} \mathrm{Cl}_{2}$ & $\mathrm{CuI}$ & 79 & 70.6 & 2.27 & 26.1 & 2.48 \\
P(bgDPP-MeOT2) & $\left.\mathrm{Pd}_{(\mathrm{PPh}}\right)_{2} \mathrm{Cl}_{2}$ & $\mathrm{CuI}$ & 84 & 61.7 & 2.37 & 30.1 & 2.62 \\
P(lgDPP-MeOT2) & $\mathrm{Pd}\left(\mathrm{PPh}_{3}\right)_{2} \mathrm{Cl}_{2}$ & $\mathrm{CuI}$ & $26^{\mathrm{c}}$ & 64.9 & 2.13 & 29.9 & 2.39 \\
\hline
\end{tabular}

${ }^{a}$ Using chloroform as the eluent. ${ }^{b}$ Using HFIP as the eluent. ${ }^{c}$ Low yield because most of the polymers is insoluble.

Table S2. Optical and Electrochemical Properties of the DPP Polymers.

\begin{tabular}{|c|c|c|c|c|c|c|c|c|}
\hline Polymer & $\begin{array}{c}E_{\text {onset }^{\mathrm{a}}} \\
{[\mathrm{V}]}\end{array}$ & $\begin{array}{l}\mathrm{IP}^{\mathrm{a}} \\
{[\mathrm{eV}]}\end{array}$ & $\begin{array}{l}\mathrm{EA}^{\mathrm{a}} \\
{[\mathrm{eV}]}\end{array}$ & $\begin{array}{c}E_{\text {onset }^{\mathrm{b}}} \\
{[\mathrm{V}]}\end{array}$ & $\begin{array}{c}\mathrm{HOMO}^{\mathrm{c}} \\
{[\mathrm{eV}]}\end{array}$ & $\begin{array}{c}E_{\mathrm{g}, \mathrm{DFT}}{ }^{\mathrm{c}} \\
{[\mathrm{eV}]}\end{array}$ & $\begin{array}{c}E_{\mathrm{g}, \mathrm{Opt}}{ }^{\mathrm{d}} \\
{[\mathrm{eV}]}\end{array}$ & $\begin{array}{l}\lambda_{\text {onset }^{\mathrm{d}}} \\
{[\mathrm{nm}]}\end{array}$ \\
\hline P(bgDPP-T) & 0.55 & 4.98 & 3.69 & 0.58 & -4.93 & 1.69 & 1.42 & 875 \\
\hline P(bgDPP-T2) & 0.48 & 4.91 & 3.74 & 0.40 & -4.92 & 1.74 & 1.37 & 906 \\
\hline P(bgDPP-MeOT2) & 0.19 & 4.62 & 3.72 & 0.23 & -4.53 & 1.50 & 1.17 & 1062 \\
\hline P(lgDPP-MeOT2) & -0.08 & 4.35 & 3.75 & 0.02 & -4.53 & 1.50 & 1.07 & 1163 \\
\hline
\end{tabular}

${ }^{\text {a }}$ Determined by the $\mathrm{CV}$ of the polymer film on ITO coated glass substrates in acetonitrile with $0.1 \mathrm{M}[n$ $\left.\mathrm{Bu}_{4} \mathrm{~N}\right]\left[\mathrm{PF}_{6}\right]$ as the supporting electrolyte. ${ }^{\mathrm{b}} 0.1 \mathrm{M} \mathrm{NaCl}$ aqueous solution as the supporting electrolyte. ${ }^{\mathrm{c}}$ Calculated results at the B3LYP/6-311G(d,p) level. ${ }^{\mathrm{d}}$ Obtained from the UV-Vis-NIR absorption spectra.

Table S3. Comparison of the OECT Performances for Polythiophene and D-A Type Polymers.

\begin{tabular}{cccccccc}
\hline Polymer & $\begin{array}{c}\mathrm{D}-\mathrm{A} \text { polymer } \\
\mathrm{Y}(\mathrm{es}) / \mathrm{N}(\mathrm{o})\end{array}$ & $\begin{array}{c}\mu \\
{\left[\mathrm{cm}^{2} \mathrm{~V}^{-1} \mathrm{~s}^{-1}\right]}\end{array}$ & $\begin{array}{c}\mathrm{C}^{*} \\
{\left[\mathrm{~F} \mathrm{~cm}^{-3}\right]}\end{array}$ & $\begin{array}{c}\mu C^{*} \\
{\left[\mathrm{~F} \mathrm{~cm}^{-1} \mathrm{~V}^{-1} \mathrm{~s}^{-1}\right]}\end{array}$ & $\begin{array}{c}\tau_{\text {on }} \\
{[\mathrm{ms}]}\end{array}$ & $\begin{array}{c}\tau_{\text {off }} \\
{[\mathrm{ms}]}\end{array}$ & $\begin{array}{c}\text { Reference } \\
\text { P(g2T-T) }\end{array}$ \\
P(g2T-TT) & $\mathrm{N}$ & $0.28 \pm 0.10$ & $220 \pm 30$ & $135 \pm 9$ & $1.4^{\mathrm{a}}$ & $1.4^{\mathrm{a}}$ & 1,2 \\
PEDOT: PSS & $\mathrm{N}$ & $0.94 \pm 0.25$ & 297 & $261 \pm 29$ & $0.42^{\mathrm{a}}$ & $0.043^{\mathrm{a}}$ & 3,4 \\
PTHS+EG & $\mathrm{N}$ & $1.9 \pm 1.3$ & $39 \pm 3$ & $47 \pm 6$ & $\mathrm{~N}^{\mathrm{e}}$ & $0.102^{\mathrm{a}}$ & 5 \\
PIBET-AO & $\mathrm{Y}$ & $\mathrm{N} / \mathrm{A}^{\mathrm{e}}$ & $\mathrm{N} / \mathrm{A}^{\mathrm{e}}$ & $5.4^{\mathrm{f}}$ & $590^{\mathrm{c}}$ & $390^{\mathrm{c}}$ & 8 \\
BBL & $\mathrm{Y}$ & $7 \times 10^{-4}$ & $930 \pm 40$ & $0.65 \pm 0.028^{\mathrm{g}}$ & $900^{\mathrm{c}}$ & $200^{\mathrm{c}}$ & 9 \\
CPEK & $\mathrm{Y}$ & $5 \times 10^{-3 \mathrm{~h}}$ & 134 & $0.67^{\mathrm{f}}$ & $\mathrm{N}^{-3} \mathrm{~A}^{\mathrm{e}}$ & $0.137^{\mathrm{d}}$ & 10 \\
P(gPyDPP-MeOT2) & $\mathrm{Y}$ & $0.030 \pm 0.007$ & 60 & $1.8 \pm 0.42^{\mathrm{g}}$ & $0.77^{\mathrm{a}}$ & $0.46^{\mathrm{a}}$ & 11 \\
P(bgDPP-MeOT2) & $\mathrm{Y}$ & $1.63 \pm 0.14^{\mathrm{h}}$ & $120.0 \pm 2.4$ & $195 \pm 21$ & $0.516^{\mathrm{a}}$ & $0.030^{\mathrm{a}}$ & This work \\
P(IgDPP-MeOT2) & $\mathrm{Y}$ & $2.15 \pm 0.27^{\mathrm{h}}$ & $80.8 \pm 1.4$ & $174 \pm 25$ & $0.578^{\mathrm{a}}$ & $0.063^{\mathrm{a}}$ & This work \\
\hline
\end{tabular}

Time constant measurements were performed with channel geometries $(W / L)$ of ${ }^{\mathrm{a}} 100 / 10 \mu \mathrm{m},{ }^{\mathrm{b}} 5 / 10 \mu \mathrm{m},{ }^{\mathrm{c}}$ $390000 / 20 \mu \mathrm{m}$, and ${ }^{\mathrm{d}} 1000 / 40 \mu \mathrm{m}$. ${ }^{\mathrm{e}}$ Data not available in the reference. ${ }^{\mathrm{f}} \mu C^{*}$ was estimated based on the 
given transconductance and device geometries. ${ }^{\mathrm{g}} \mu C^{*}$ was calculated as the product of the measured $\mu$ and $C^{*} .{ }^{\mathrm{h}} \mu$ was calculated from the measured $\mu C^{*}$ and $C^{*}$. 
a

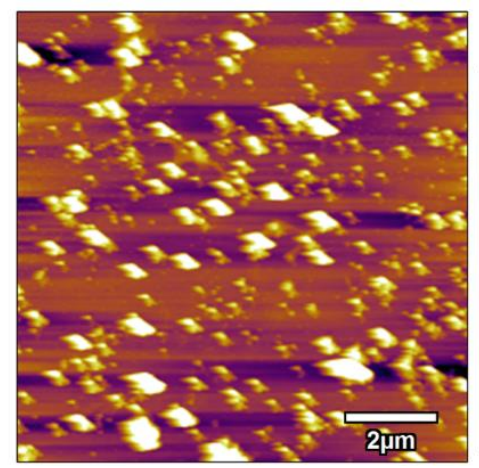

b

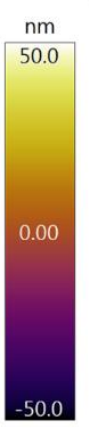

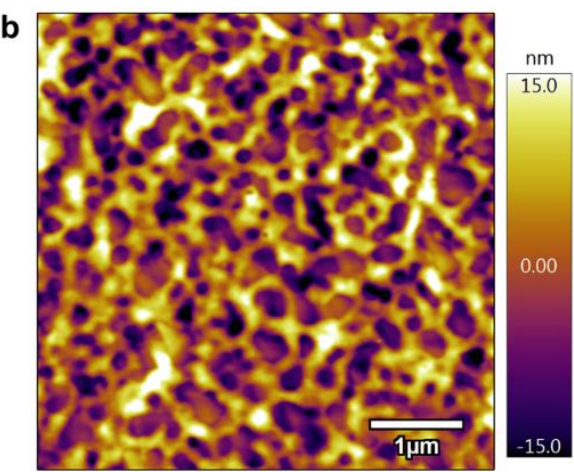

Figure S1 AFM topography images of the as-fabricated P(bgDPP-MeOT2) film. The polymer channel was fabricated by spin-coating its $3 \mathrm{mg} / \mathrm{mL}$ (a) chloroform and (b) hexafluoroisopropanol solution at $1000 \mathrm{rpm}$ for $60 \mathrm{~s}$ on a silicon dioxide substrate.
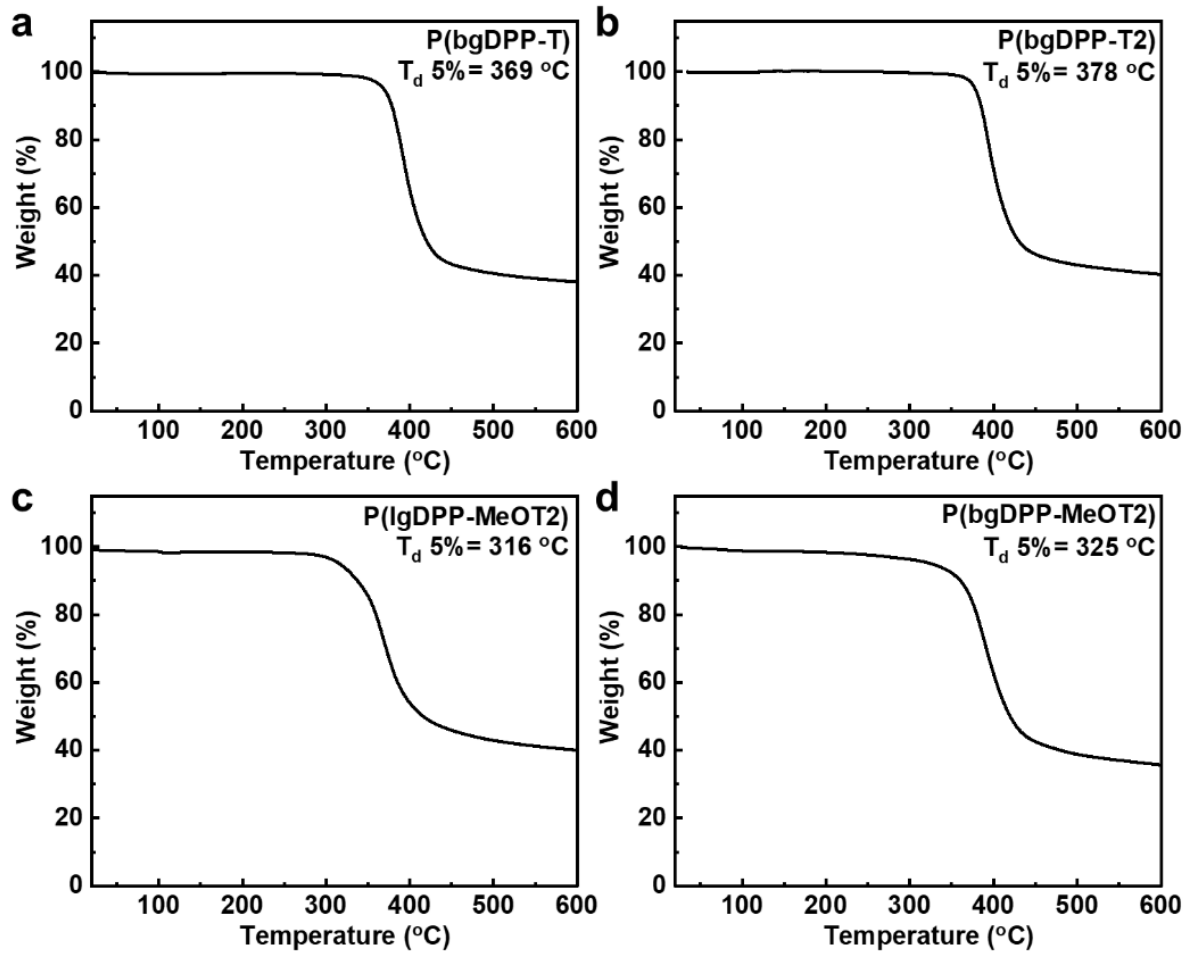

Figure S2 Thermal gravity analyses (TGA) of (a) P(bgDPP-T), (b) P(bgDPP-T2), (c) P(lgDPP-MeOT2), and (d) P(bgDPP-MeOT2). 

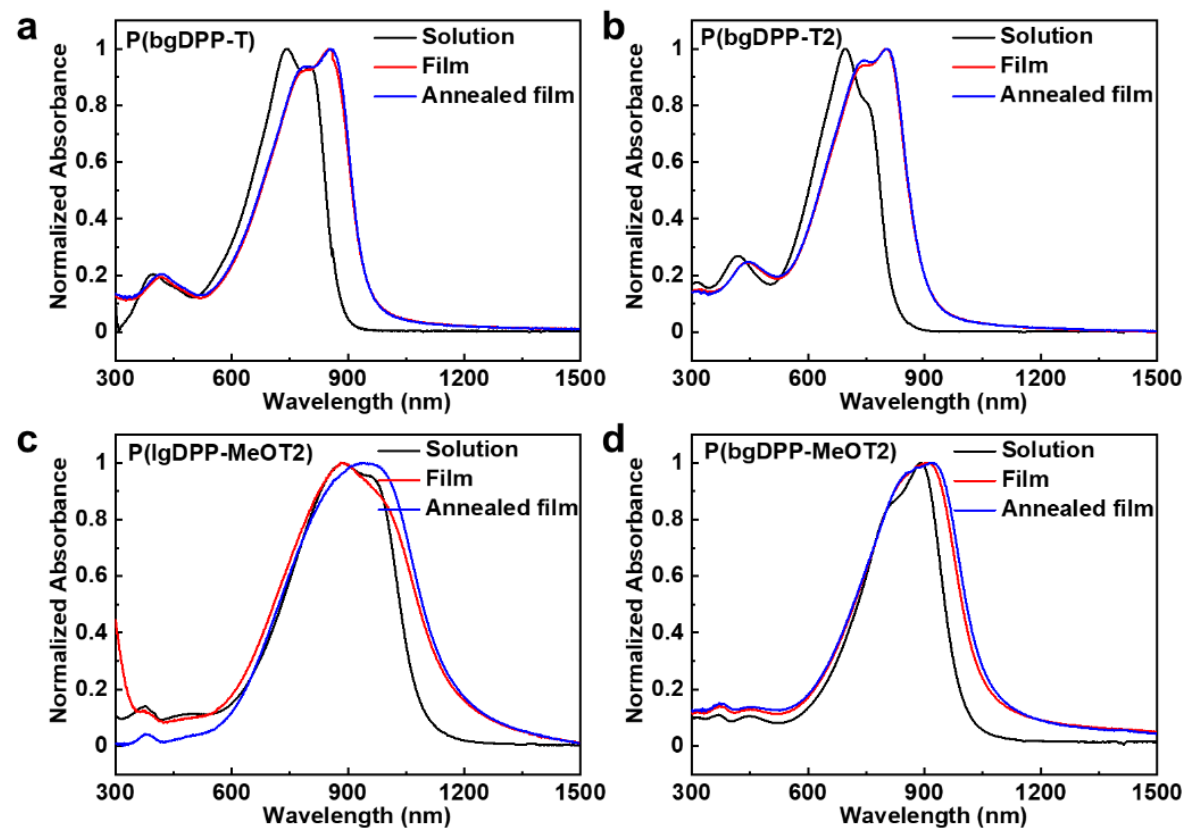

Figure S3 Normalized UV-vis-NIR absorption spectra of (a) P(bgDPP-T), (b) P(bgDPP-T2), (c) P(lgDPPMeOT2) and (d) P(bgDPP-MeOT2) in diluted chloroform $\left(1.0 \times 10^{-5} \mathrm{M}\right)$, in thin film, and in annealed thin film $\left(80^{\circ} \mathrm{C}, 10 \mathrm{~min}\right)$.
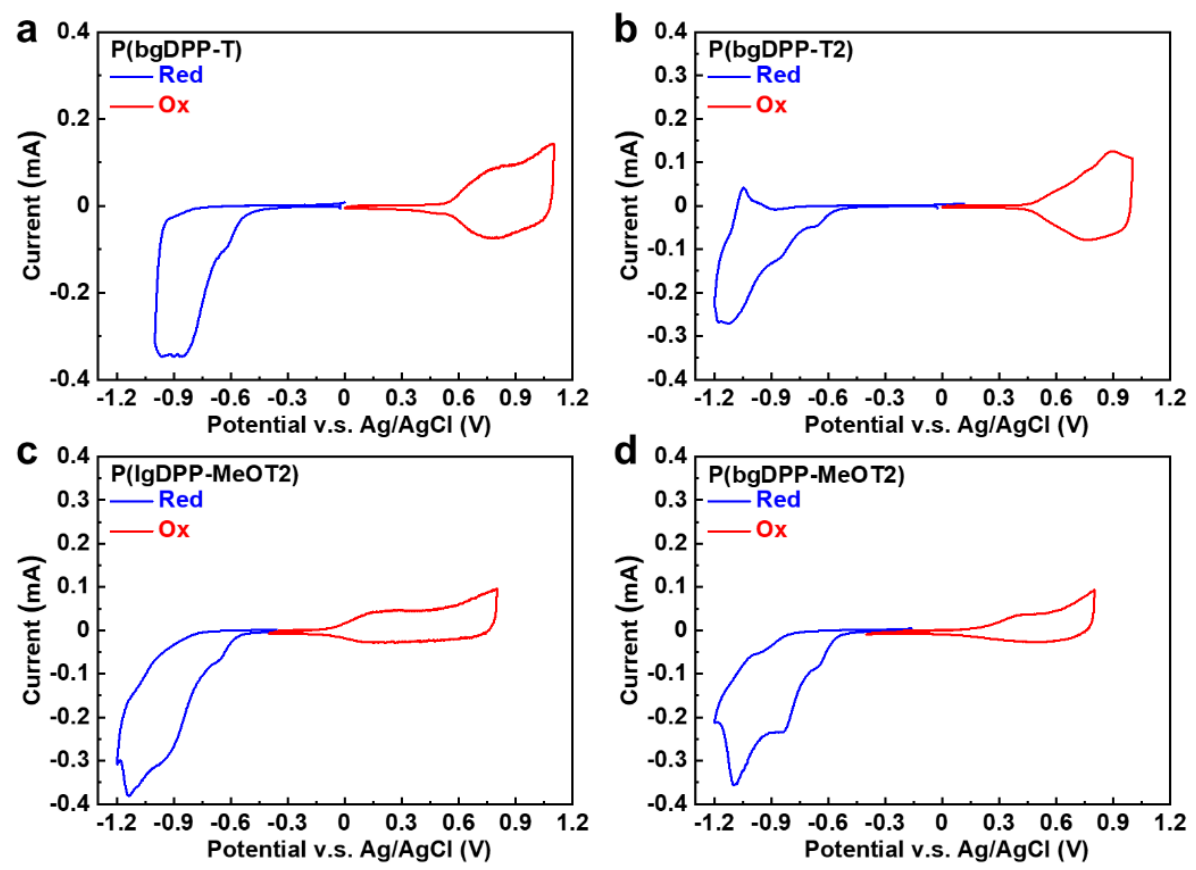

Figure S4 Cyclic voltammograms of (a) P(bgDPP-T), (b) P(bgDPP-T2), (c) P(lgDPP-MeOT2), and (d) $\mathrm{P}(\mathrm{bgDPP}-\mathrm{MeOT} 2)$ in acetonitrile solution with $0.1 \mathrm{M}$ tetrabutylammonium hexafluorophosphate as the supporting electrolyte. 

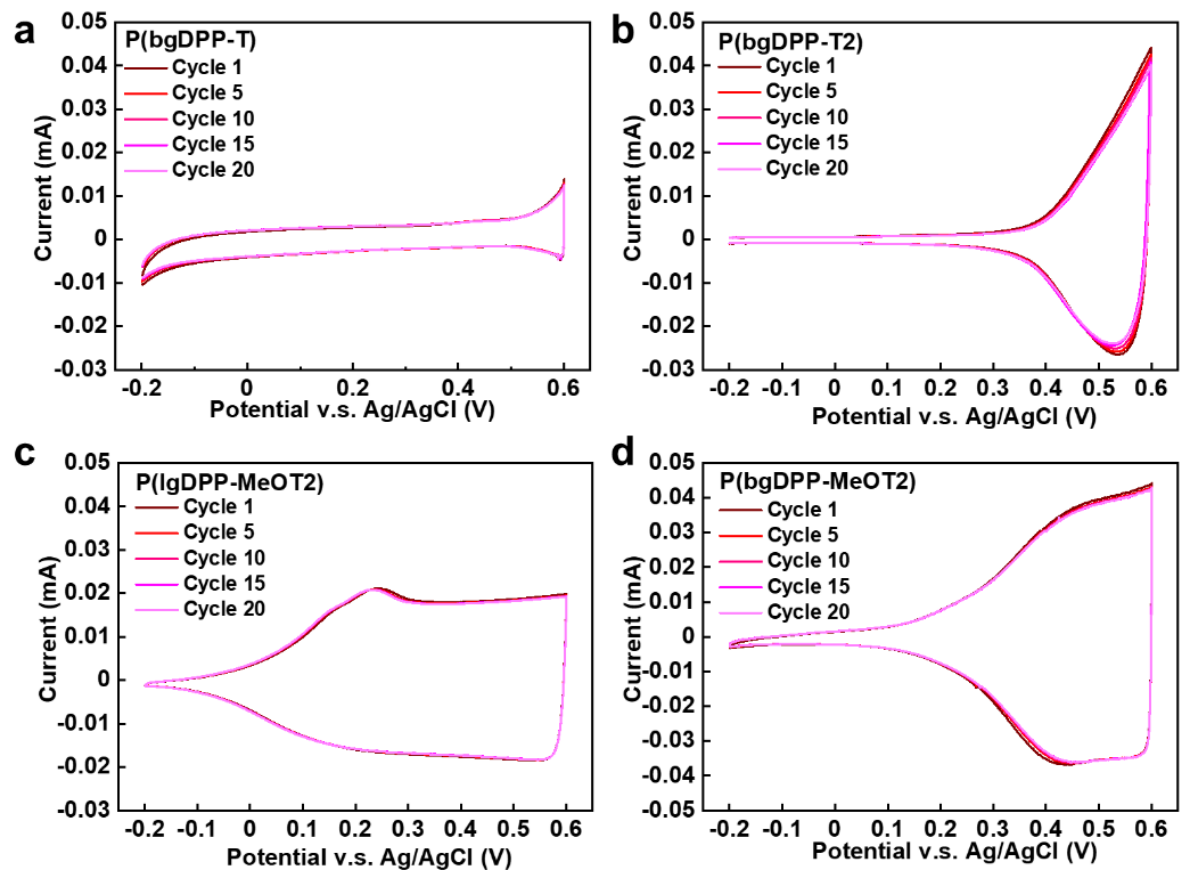

Figure S5 Cyclic voltammograms of (a) P(bgDPP-T), (b) P(bgDPP-T2), (c) P(lgDPP-MeOT2), and (d) $\mathrm{P}(\mathrm{bgDPP}-\mathrm{MeOT}$ ) in aqueous solution with $0.1 \mathrm{M}$ sodium chloride as the supporting electrolyte. All the $\mathrm{CV}$ scans were repeated for 20 cycles.

a

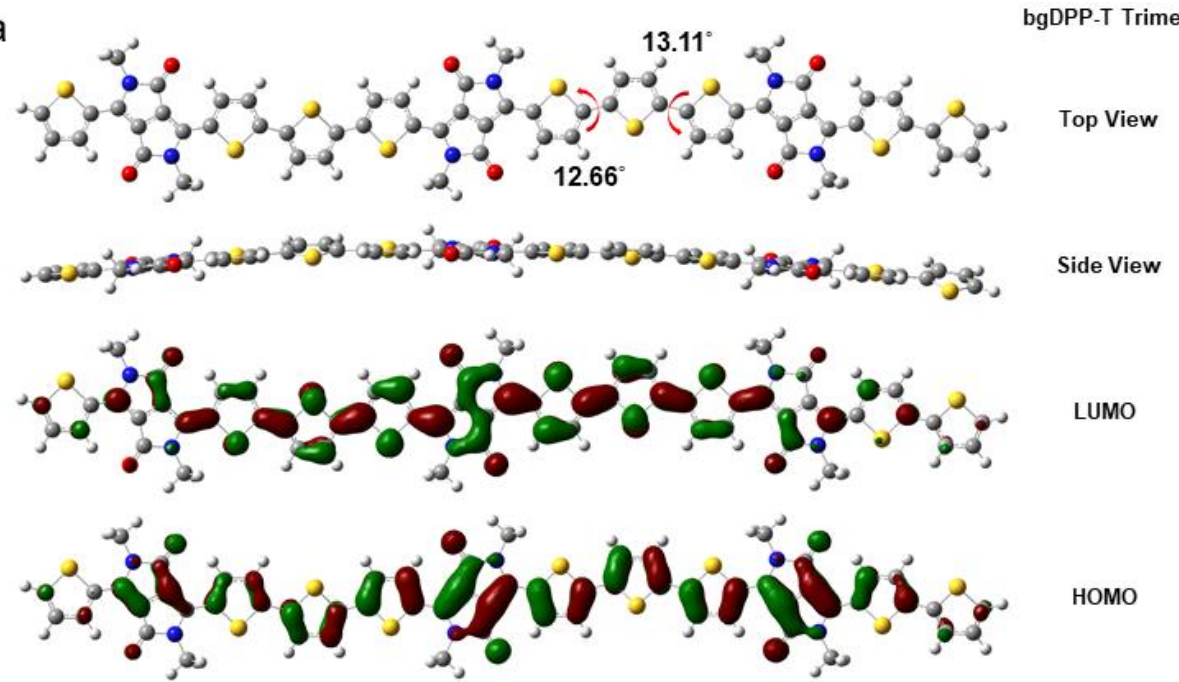


b bgDPP-T2 Trimer

b

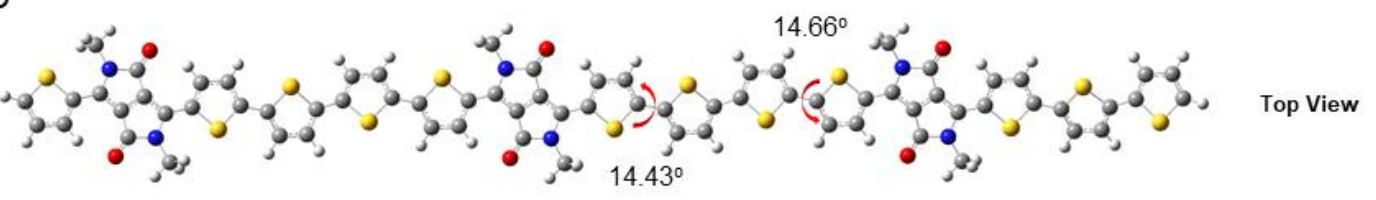

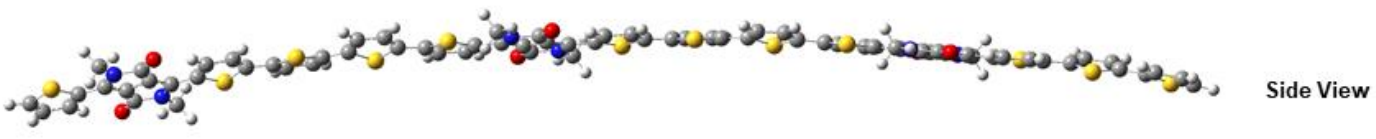
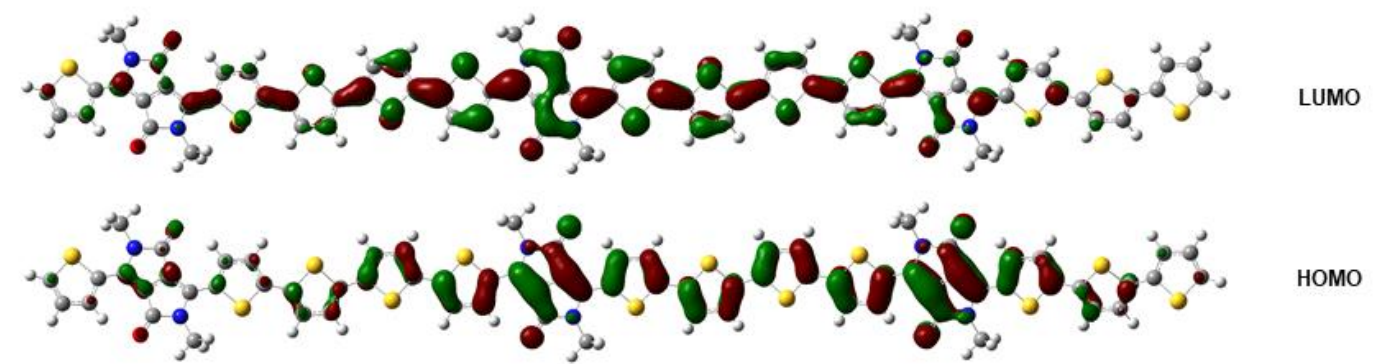

Figure S6 DFT-optimized geometries and molecular frontier orbitals of the trimer of (a) bgDPP-T and (b) bgDPP-T2. Calculations were performed at B3LYP/6-311G(d,p) level. Branched glycol side chains were replaced with methyl groups to simplify the calculation.
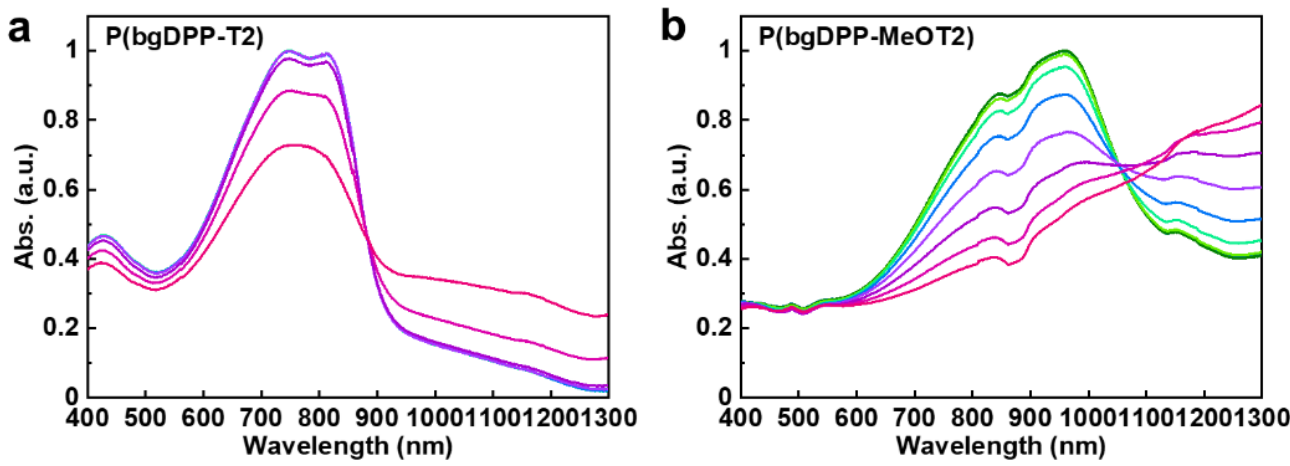

Figure S7 UV-vis-NIR spectra of (a) P(bgDPP-T) and (b) P(bgDPP-T2) upon continuously increasing the bias on the polymer film. The applied voltage ranged from $-0.2 \mathrm{~V}$ to $0.6 \mathrm{~V}$ with an interval of $0.1 \mathrm{~V}$. 

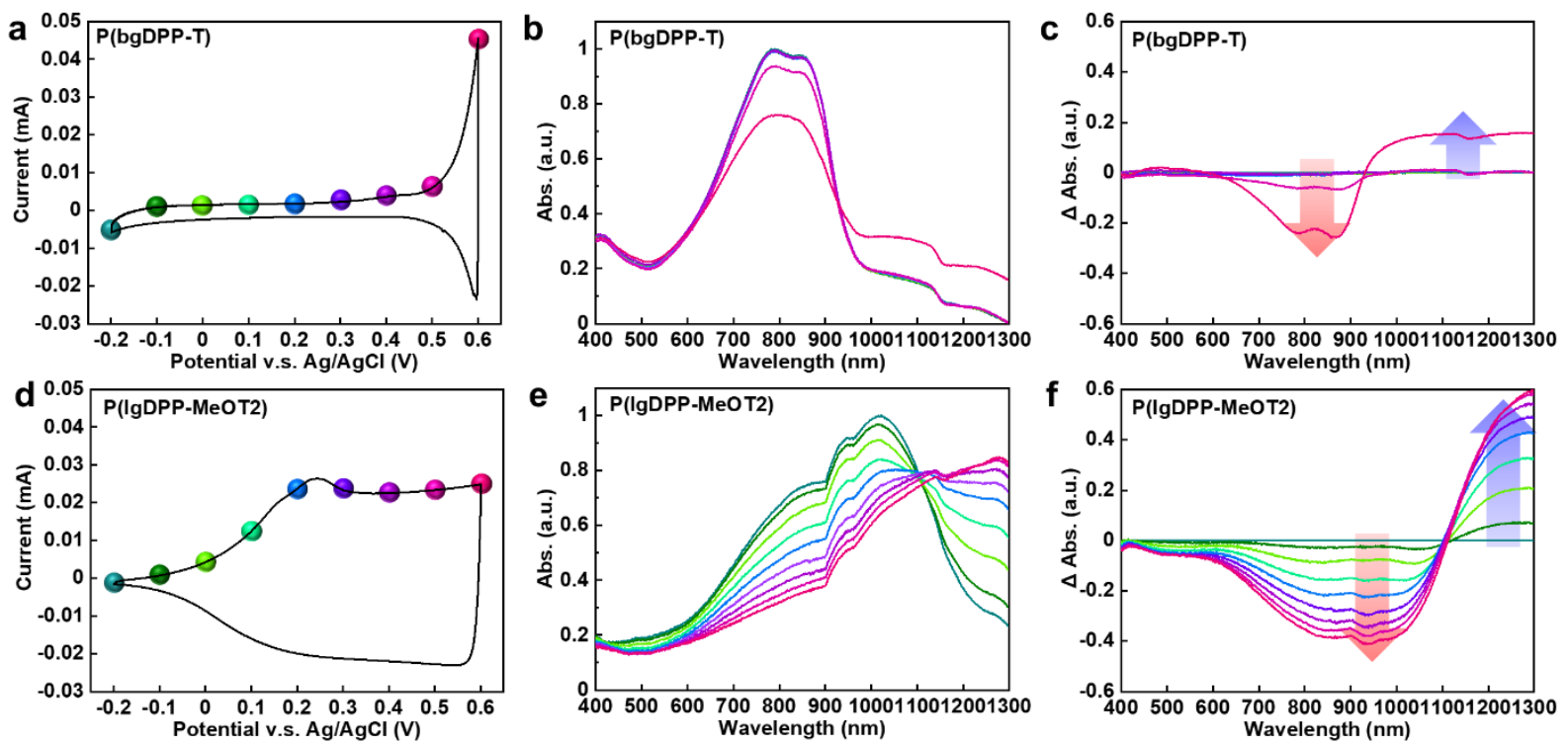

Figure S8 Cyclic voltammograms, UV-vis-NIR spectra, and the differential spectra of (a-c)P(bgDPP-T), and (d-f) P(lgDPP-MeOT2). The color-coding UV-vis-NIR spectra indicate the applied voltage on the polymer film, ranging from $-0.2 \mathrm{~V}$ to $0.6 \mathrm{~V}$ with an interval of $0.1 \mathrm{~V}$.
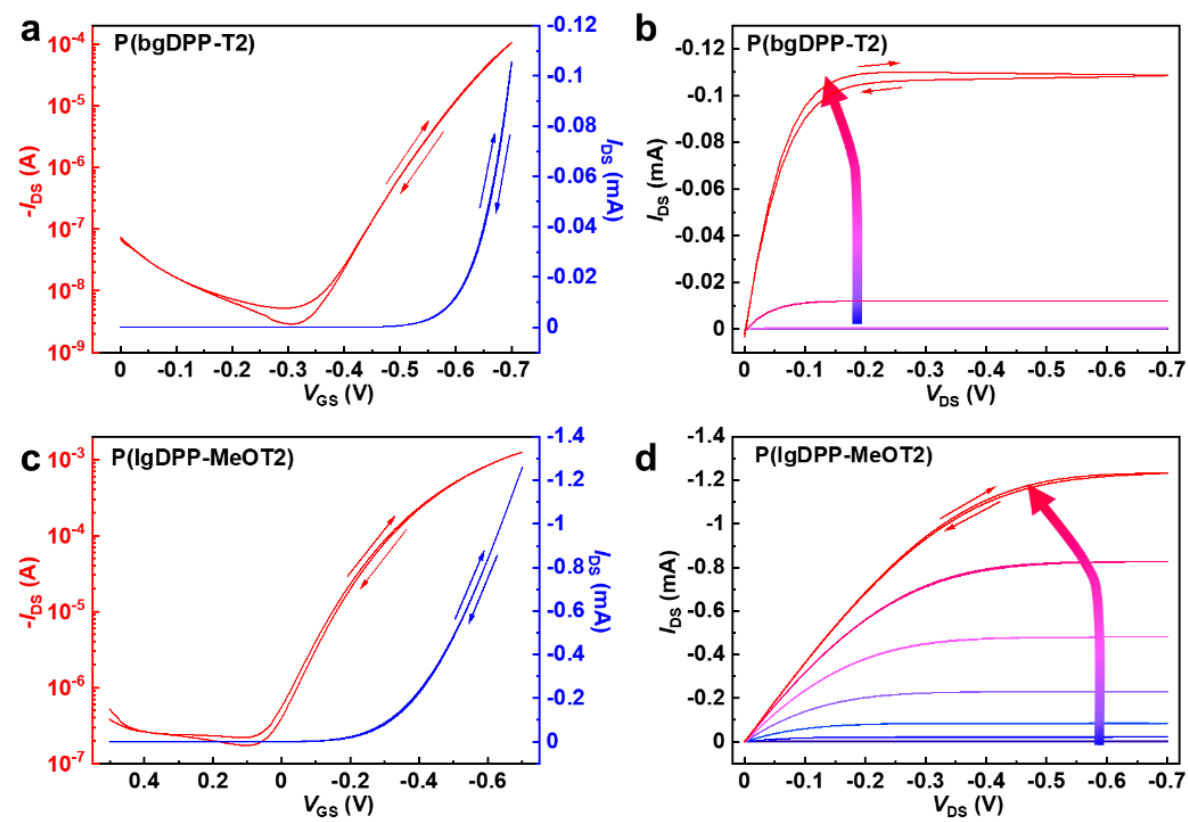

Figure S9 Transfer and output characteristics of (a-b) P(bgDPP-T2) and (c-d) P(lgDPP-MeOT2) OECTs. Channel dimensions: $W / L=1000 / 10 \mu \mathrm{m}, d=30.8 \pm 1.7 \mathrm{~nm}$ for P(bgDPP-T2), and $31.0 \pm 1.3 \mathrm{~nm}$ for P(lgDPPMeOT2). $V_{\text {DS }}$ was set to $-0.6 \mathrm{~V}$. 

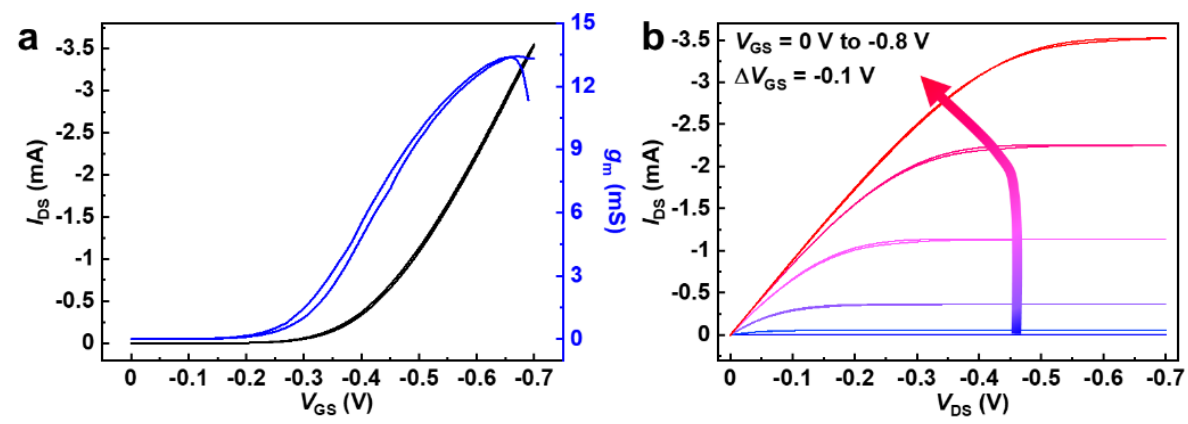

Figure S10 (a) Transfer and transconductance characteristics of P(bgDPP-MeOT2)-based OECT with a thick film, (b) The corresponding output characteristics. Channel dimensions: $W / L=1000 / 10 \mu \mathrm{m}, d=75.0 \pm 2.0$ nm. $V_{\mathrm{DS}}$ was set to $-0.6 \mathrm{~V}$.

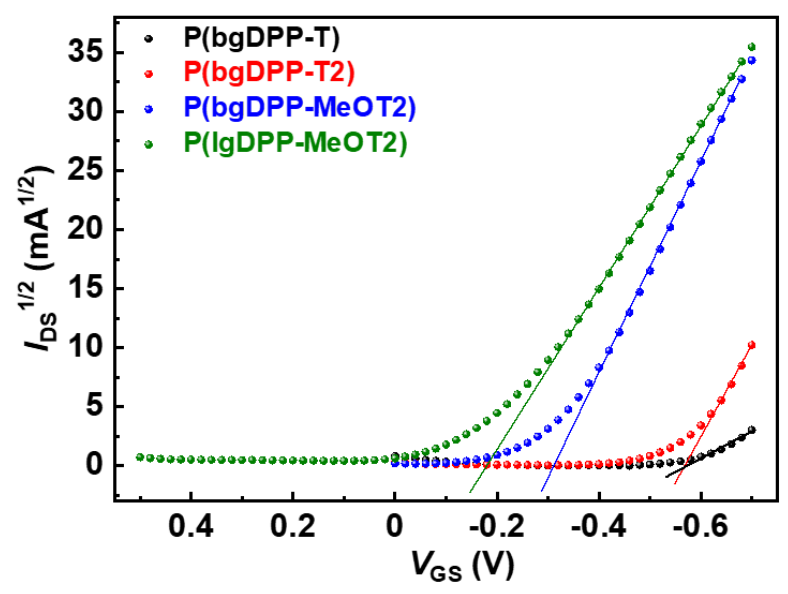

Figure S11 $I_{\mathrm{DS}}{ }^{1 / 2}$ vs. $V_{\mathrm{GS}}$ plots of the DPP polymer based OECTs. The threshold voltages, $V_{\mathrm{Th}}$, were determined by extrapolating the linear region of the curves. Channel dimensions: $W / L=1000-10 \mu \mathrm{m}, d=$ $29.0 \pm 0.8 \mathrm{~nm}$ for $\mathrm{P}(\mathrm{bgDPP}-\mathrm{T}), 30.8 \pm 1.7 \mathrm{~nm}$ for $\mathrm{P}(\mathrm{bgDPP}-\mathrm{T} 2)$, $35.2 \pm 1.7 \mathrm{~nm}$ for $\mathrm{P}(\mathrm{bgDPP}-\mathrm{MeOT} 2)$, and $31.0 \pm 1.3 \mathrm{~nm}$ for $\mathrm{P}(\operatorname{lgDPP}-\mathrm{MeOT} 2)$. $V_{\mathrm{DS}}$ was set to $-0.6 \mathrm{~V}$. 


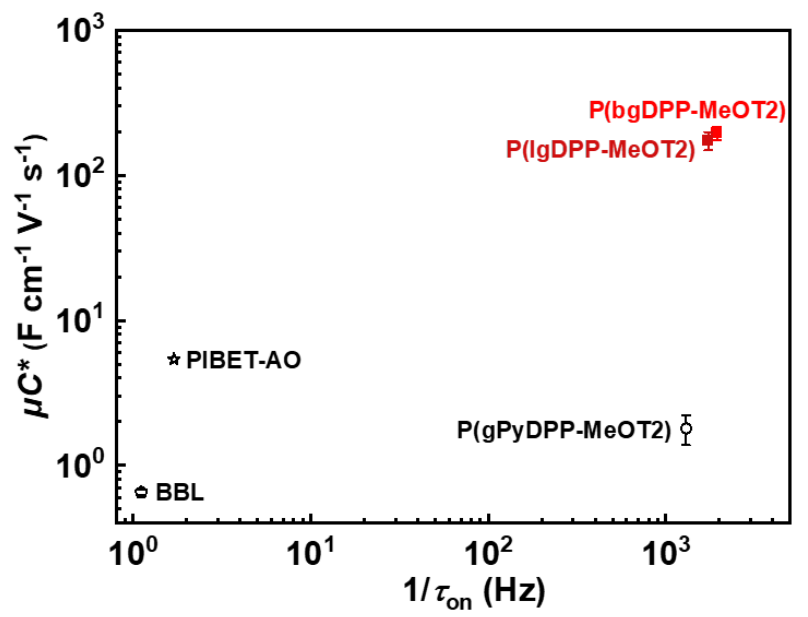

Figure S12 Performance comparison of the $\mu C^{*}-1 / \tau_{\text {on }}$ plot for $\mathrm{P}(\operatorname{bgDPP}-\mathrm{MeOT} 2), \mathrm{P}(\operatorname{lgDPP}-\mathrm{MeOT} 2)$ and other reported D-A polymers.

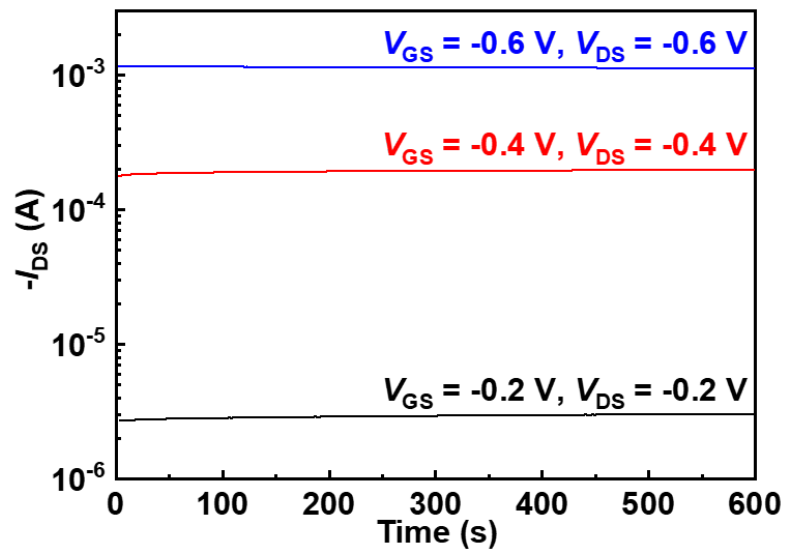

Figure S13 Continuous stressing of a P(bgDPP-MeOT2) OECT device upon the indicated $V_{\mathrm{DS}}$ and $V_{\mathrm{GS}}$ values. 

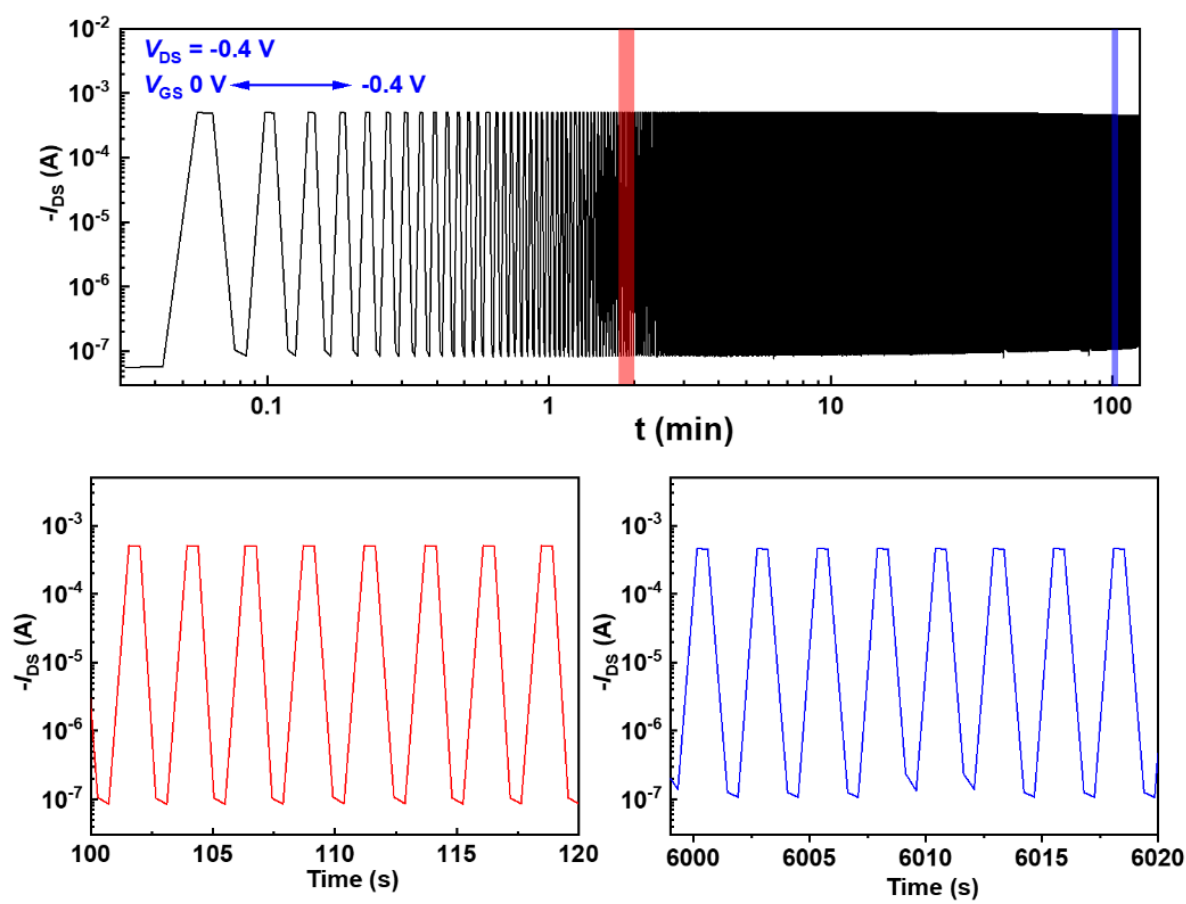

Figure S14 The enlargement view of the on-off switching plot of P(bgDPP-MeOT2) OECT device at two different time zones, highlighted with red and blue boxes. Switching on time of $V_{G S}$ and the interval time were set as $2 \mathrm{~s}$ both.

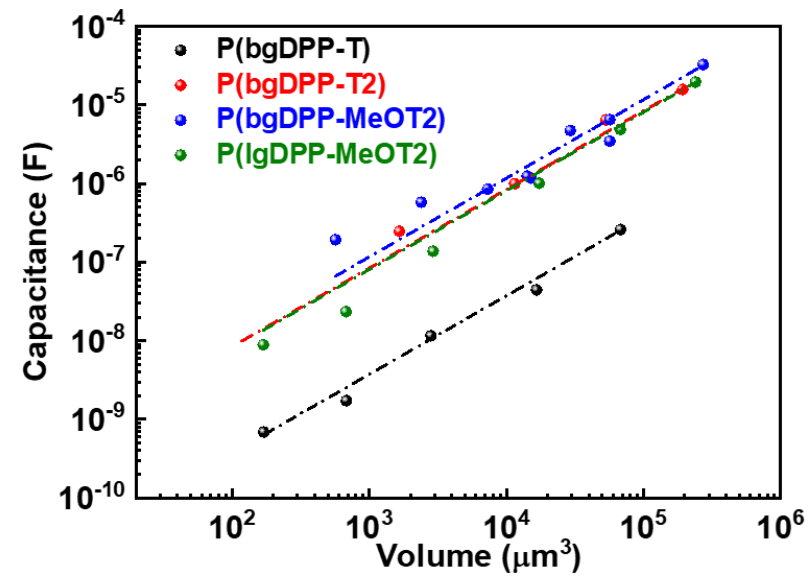

Figure S15 Capacitance-volume relationship of the DPP polymers. All the data were measured through the electrochemical impedance spectroscopy method. Linear fitting was performed to obtain their corresponding volumetric capacitance. 

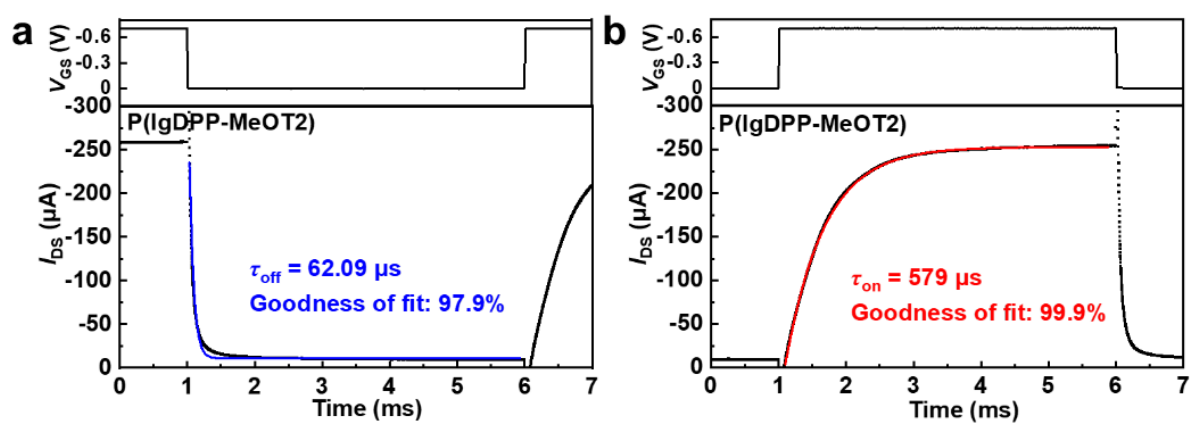

Figure S16 (a, b) Off- \& on-time constant of P(lgDPP-MeOT2) obtained by applying a gate voltage pulse with a time scale of $5 \mathrm{~ms}$. Blue and red lines were fitted through exponential decay function. $W / L=100 / 10$ $\mu \mathrm{m}$ and $d=31.7 \pm 1.0 \mathrm{~nm}$. $V_{\mathrm{DS}}$ was set to $-0.6 \mathrm{~V}$.
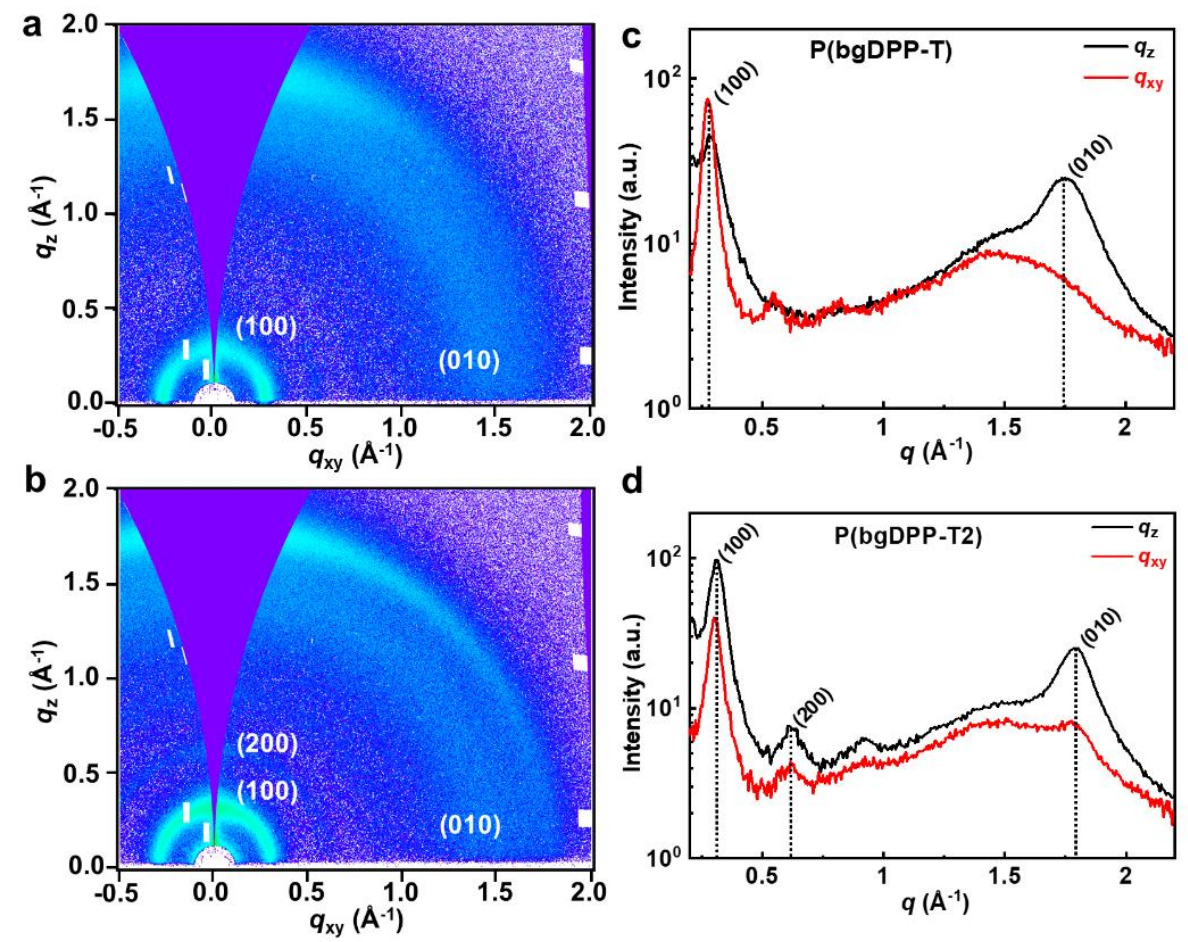

Figure S17 2D GIWAXS patterns of (a) P(bgDPP-T), and (b) P(bgDPP-T2). (c-d) The corresponding line cuts of P(bgDPP-T) and P(bgDPP-T2). Cuts along the $q_{\mathrm{xy}}$ direction (red) represent the scattering from the inplane, while the scattering in the $q_{\mathrm{z}}$ direction (black) results from the out-of-plane. The associated lamellar ( $h 00)$, and $\pi-\pi$ stacking $(0 k 0)$ peaks are indicated. 


\section{Synthetic Procedure and Characterization}

Scheme S1. Synthesis of the monomers and polymers.

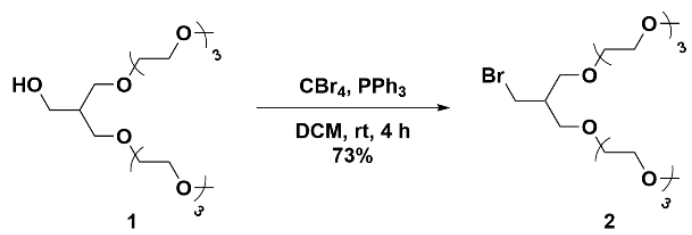<smiles>COc1cc([Si](C)(C)C)sc1-c1sc([Si](C)(C)C)cc1OC</smiles>

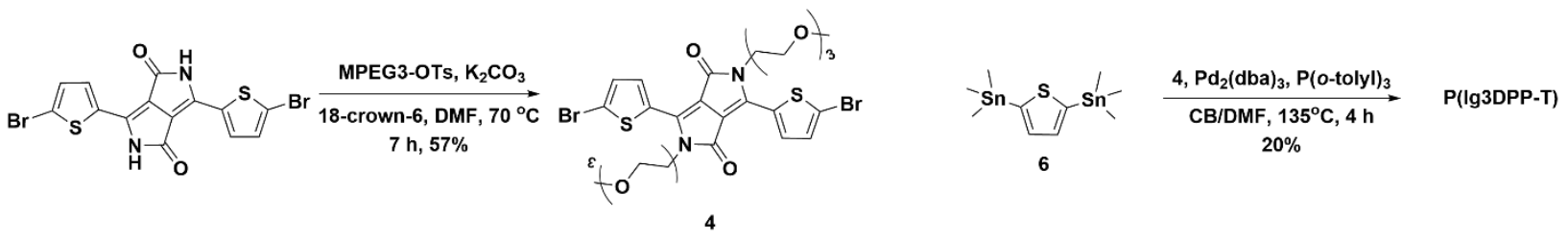

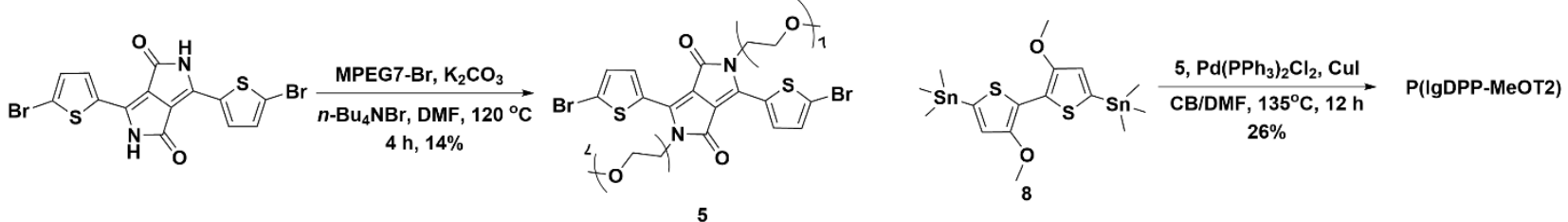

Compound $\mathbf{1}$ was synthesized according to the reported procedure. ${ }^{12}$
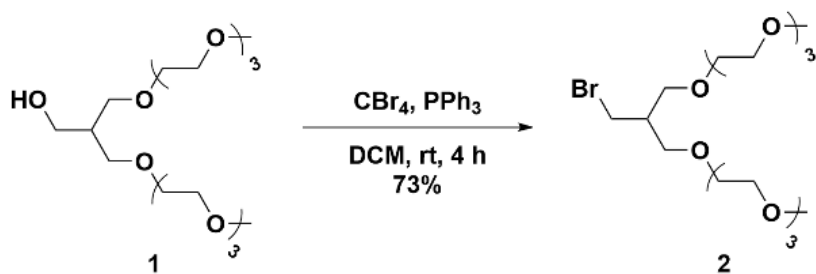

Synthesis of 2: To a $25 \mathrm{~mL}$ two-necked round-bottom flask, 1 (500 mg, $1.26 \mathrm{mmol}$ ), carbon tetrabromide (461 mg, $1.39 \mathrm{mmol})$, and $5 \mathrm{~mL}$ DCM were added. Then triphenylphosphine (362 mg, $1.38 \mathrm{mmol})$ in $5 \mathrm{~mL}$ DCM was added slowly. The mixture was stirred at room temperature for 4 hours. The resulting mixture was sent to rotary evaporation, and the solvent was removed. The residue was purified through silica gel chromatography $($ DCM/ethyl acetate $(E A)=1 / 1)$ to afford 2 as a colorless liquid $(425 \mathrm{mg}$, yield $73 \%) .{ }^{1} \mathrm{H}$ 
$\operatorname{NMR}\left(400 \mathrm{MHz}, \mathrm{CDCl}_{3}\right) \delta 3.68-3.45(\mathrm{~m}, 30 \mathrm{H}), 3.39(\mathrm{~s}, 6 \mathrm{H}), 2.27(\mathrm{~m}, 1 \mathrm{H}) \cdot{ }^{13} \mathrm{C} \mathrm{NMR}\left(101 \mathrm{MHz}, \mathrm{CDCl}_{3}\right) \delta$ 71.9, 70.6, 70.6, 70.5, 70.4, 70.0, 59.0, 41.3, 33.5. MALDI-TOF HRMS calcd. for $\left[\mathrm{M}+\mathrm{NH}_{4}\right]^{+}: 461.1750$; found: 461.1734 .

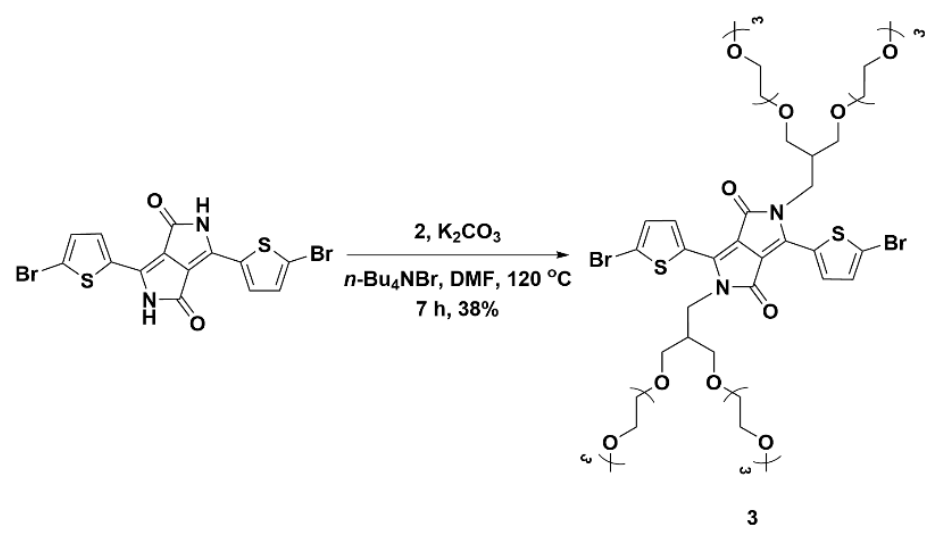

Synthesis of 3: A $25 \mathrm{~mL}$ Schlenk tube was charged with 2 (440 mg, $0.95 \mathrm{mmol})$, 3,6-bis(5-bromothiophen2-yl)-2,5-dihydropyrrolo[3,4-c]pyrrole-1,4-dione (DPP) (200 mg, $0.44 \mathrm{mmol}$ ), potassium carbonate (88 mg, $0.436 \mathrm{mmol}$ ), tetrabutylammonium bromide (300 $\mathrm{mg}, 0.93 \mathrm{mmol}$ ), and $5 \mathrm{~mL}$ dry DMF. The resulting mixture was heated to $120^{\circ} \mathrm{C}$ and stirred for $7 \mathrm{~h}$. Then the reaction mixture was cooled to room temperature and poured into water. The mixture was extracted with DCM with three times and washed with brine. Then the solvent was removed under reduced pressure, and the residue was purified by silica gel chromatography $(\mathrm{DCM} / \mathrm{methanol}=60 / 1)$ to afford $\mathbf{3}(201 \mathrm{mg}$, yield $38 \%)$ as a purplish red liquid. ${ }^{1} \mathrm{H}$ NMR $\left(400 \mathrm{MHz}, \mathrm{CDCl}_{3}\right)$ $\delta 8.54(\mathrm{~d}, J=4.2 \mathrm{~Hz}, 2 \mathrm{H}), 7.22(\mathrm{~d}, J=4.2 \mathrm{~Hz}, 2 \mathrm{H}), 4.12(\mathrm{~d}, J=7.4 \mathrm{~Hz}, 4 \mathrm{H}), 3.63(\mathrm{~m}, 56 \mathrm{H}), 3.36(\mathrm{~s}, 12 \mathrm{H})$, $2.43(\mathrm{~m}, 2 \mathrm{H}) .{ }^{13} \mathrm{C}$ NMR $\left(101 \mathrm{MHz}, \mathrm{CDCl}_{3}\right) \delta 161.2,134.7,131.4,131.2,119.2,107.7,71.8,70.6,70.5,70.4$, 70.3, 70.0, 58.9, 41.6, 40.1. MALDI-TOF HRMS calcd. for [M + $\left.\mathrm{NH}_{4}\right]^{+}:$1234.3396; found: 1234.3406.

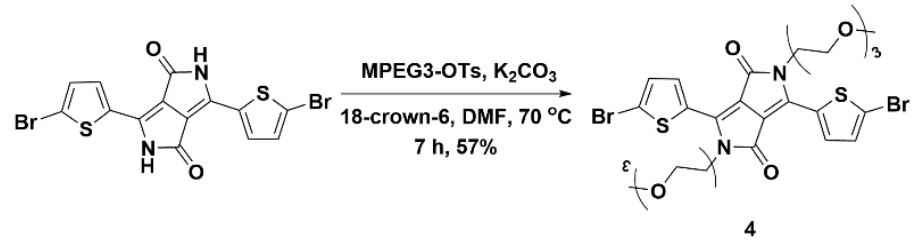

Synthesis of 4: Under nitrogen atmosphere, to a $250 \mathrm{~mL}$ two-necked round-bottom flask, DPP (500 mg, 1.66 mmol), triethylene glycol monomethyl 4-methyl-benzenesulfonicacimethyl ester (MPEG3-OTs, $1.163 \mathrm{~g}$, $3.66 \mathrm{mmol})$, potassium carbonate $(176 \mathrm{mg}, 1.66 \mathrm{mmol}), 18$-crown-6 (5 mg, $0.02 \mathrm{mmol})$ and $30 \mathrm{~mL}$ anhydrous DMF were added. The mixture was heated and stirred under $70{ }^{\circ} \mathrm{C}$ for 8 hours. Then the mixture was poured 
to water and exacted with DCM. The organic phase was dried with $\mathrm{Na}_{2} \mathrm{SO}_{4}$ and sent to a rotatary evaporator to remove the solvent. The residue was purified through silica chromatography (petroleum ether $(\mathrm{PE}) / \mathrm{EA}=$ 3/1) to afford 4 as a dark red solid (574 mg, yield 57\%). ${ }^{1} \mathrm{H}$ NMR (400 MHz, $\left.\mathrm{CDCl}_{3}\right) \delta 8.48(\mathrm{~d}, J=4.2 \mathrm{~Hz}$, 2H), $7.20(\mathrm{~d}, J=4.2 \mathrm{~Hz}, 2 \mathrm{H}), 4.16(\mathrm{t}, J=5.9 \mathrm{~Hz}, 4 \mathrm{H}), 3.77(\mathrm{t}, J=5.9 \mathrm{~Hz}, 4 \mathrm{H}), 3.66-3.53(\mathrm{~m}, 12 \mathrm{H}), 3.52-$ $3.45(\mathrm{~m}, 4 \mathrm{H}), 3.34(\mathrm{~s}, 6 \mathrm{H}) .{ }^{13} \mathrm{C} \mathrm{NMR}\left(101 \mathrm{MHz}, \mathrm{CDCl}_{3}\right) \delta 161.2,139.5,134.8,131.4,131.1,119.3,107.9$, 71.9, 70.8, 70.5, 70.5, 68.9, 59.0, 42.2. MALDI-TOF HRMS calcd. for $\left[\mathrm{M}+\mathrm{NH}_{4}\right]^{+}:$766.0463; found: 766.0462 .

Synthesis of 5

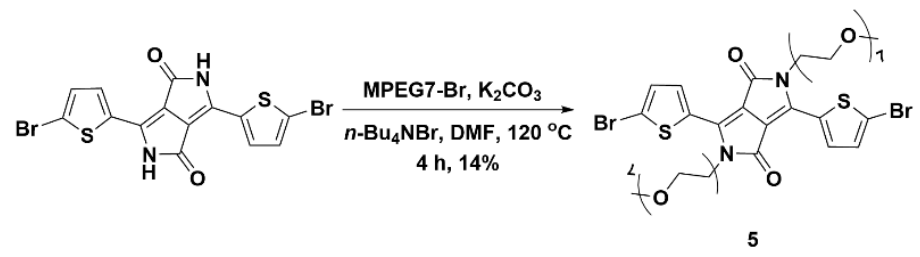

Under nitrogen atmosphere, to a $250 \mathrm{~mL}$ two-necked round-bottom flask, DPP (1.00 g, $2.18 \mathrm{mmol})$, methoxy hepta(ethylene glycol)bromide (MPEG7-Br, $1.101 \mathrm{~g}, 5.46 \mathrm{mmol}$ ), potassium carbonate (578 mg, $5.46 \mathrm{mmol}$ ), tetrabutylammonium bromide $(1.40 \mathrm{~g}, 4.36 \mathrm{mmol})$, and $30 \mathrm{~mL}$ anhydrous DMF were added. The resulting mixture was heated to $120^{\circ} \mathrm{C}$ and stirred for $4 \mathrm{~h}$. Then the reaction mixture was cooled to room temperature and poured into water. The mixture was extracted with DCM three times and washed with brine. Then the solvent was removed under reduced pressure, the residue was purified by silica gel chromatography $(\mathrm{DCM} / \mathrm{methanol}=40 / 1)$ to afford $5(351 \mathrm{mg}$, yield $14 \%)$ as a purplish-red solid. ${ }^{1} \mathrm{H}$ NMR $\left(400 \mathrm{MHz}, \mathrm{CDCl}_{3}\right)$ $\delta 8.46(\mathrm{~d}, J=4.2 \mathrm{~Hz}, 2 \mathrm{H}), 7.18(\mathrm{~d}, J=4.2 \mathrm{~Hz}, 2 \mathrm{H}), 4.14(\mathrm{t}, J=5.9 \mathrm{~Hz}, 4 \mathrm{H}), 3.74(\mathrm{t}, J=5.9 \mathrm{~Hz}, 4 \mathrm{H}), 3.67-$ 3.49 (m, 48H), 3.35 (s, 6H). ${ }^{13} \mathrm{C} \mathrm{NMR}\left(101 \mathrm{MHz}, \mathrm{CDCl}_{3}\right) \delta 161.1,134.8,131.3,131.0,119.2,107.9,70.7$, 70.5, 70.5, 70.4, 68.8, 58.9, 42.1. MALDI-TOF HRMS calcd. for $\left[\mathrm{M}+\mathrm{NH}_{4}\right]^{+}:$1118.2559; found: 1118.2559 .

\section{General Procedure for Polymerization:}

To a $25 \mathrm{~mL}$ Schlenk tube, tin reagent (1.00 eq.), DPP monmer (1.00 eq.), Pd catalyst (0.04 eq.), CuI (0.04 eq.), anhydrous DMF, and anhydrous chlorobenzene were added under nitrogen atmosphere. The tube was charged with nitrogen through a freeze-pump-thaw cycle for three times. The sealed tube was heated to 135 ${ }^{\circ} \mathrm{C}$ and stirred for a given time (Scheme S1). After cooling the reaction mixture to room temperature, diethylphenylazothioformamide $(3 \mathrm{mg}$ ) was added to remove the catalyst and the resulting mixture was 
stirred at $80{ }^{\circ} \mathrm{C}$ for $1 \mathrm{~h}$. The reaction mixture was poured into $20 \mathrm{~mL}$ hexane to precipitate the polymer and filtered. The polymer solid was placed in a Soxhlet extractor and extracted with hexane, methanol, acetone, and chloroform. The chloroform solution was concentrated under reduced pressure and then poured into 20 $\mathrm{mL}$ hexane to re-precipitate the polymer. The suspension was filtered and dried in vacuum to afford the polymer.

P(lg3DPP-T): Dark green solid (yield: 20\%). Most of the polymer is insoluble, leading to a low yield.

P(bgDPP-T): Dark green solid (yield: 62\%). ${ }^{1} \mathrm{H}$ NMR (600 MHz, $\mathrm{CDCl}_{3}, 293 \mathrm{~K}$, ppm) $\delta 8.83,7.75,4.24$, $3.84-3.45,3.35,2.52$.

P(bgDPP-T2): Dark green solid (yield: 79\%). ${ }^{1} \mathrm{H}$ NMR (600 MHz, $\left.\mathrm{CDCl}_{3}, 293 \mathrm{~K}, \mathrm{ppm}\right) \delta 8.84,7.04,3.78-$ $3.55,3.35,2.46$

P(bgDPP-MeOT2): Dark green solid (yield: 84\%). ${ }^{1} \mathrm{H}$ NMR (600 MHz, $\left.\mathrm{CDCl}_{3}, 293 \mathrm{~K}, \mathrm{ppm}\right) \delta 8.82,7.08$, $4.30-3.45,3.35,2.54$.

P(IgDPP-MeOT2): Dark green solid (yield 26\%). Most of the polymer is insoluble, leading to a low yield. ${ }^{1} \mathrm{H}$ NMR (500 MHz, $\left.\mathrm{CDCl}_{2} \mathrm{CDCl}_{2}, 383 \mathrm{~K}, \mathrm{ppm}\right) \delta 8.68,7.35,7.06,4.43-3.43,3.31$. 


\section{4. ${ }^{1} \mathrm{H}$ and ${ }^{13} \mathrm{C}$ NMR spectra}

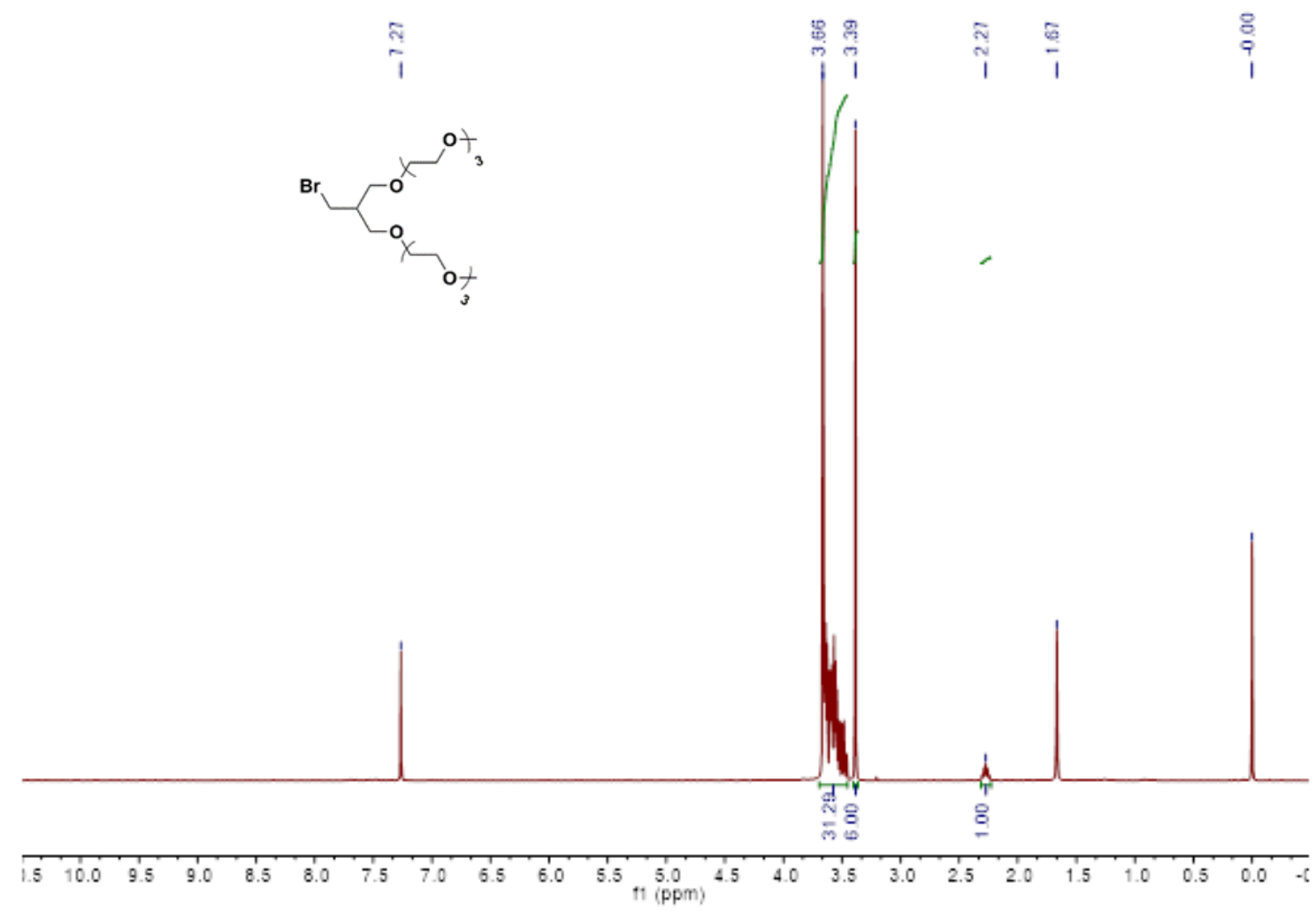

Figure S18 ${ }^{1} \mathrm{H}$ NMR spectrum of 2.

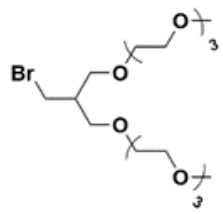

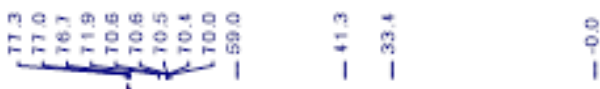

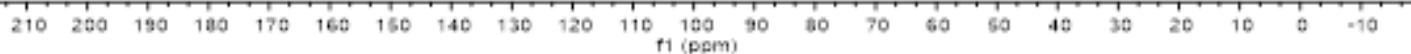

Figure S19 ${ }^{13} \mathrm{C}$ NMR spectrum of 2. 


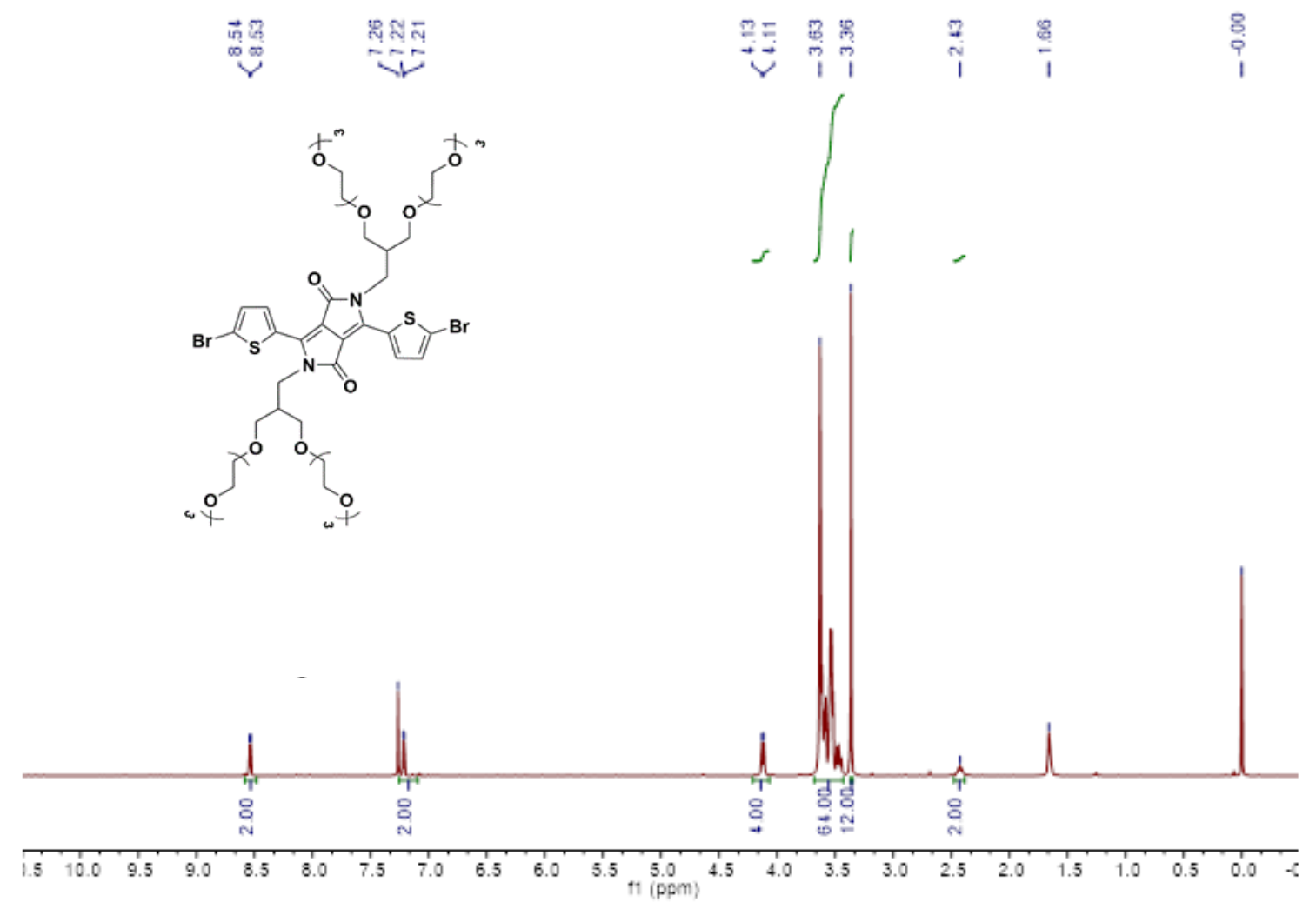

Figure S20 ${ }^{1} \mathrm{H}$ NMR spectrum of 3.

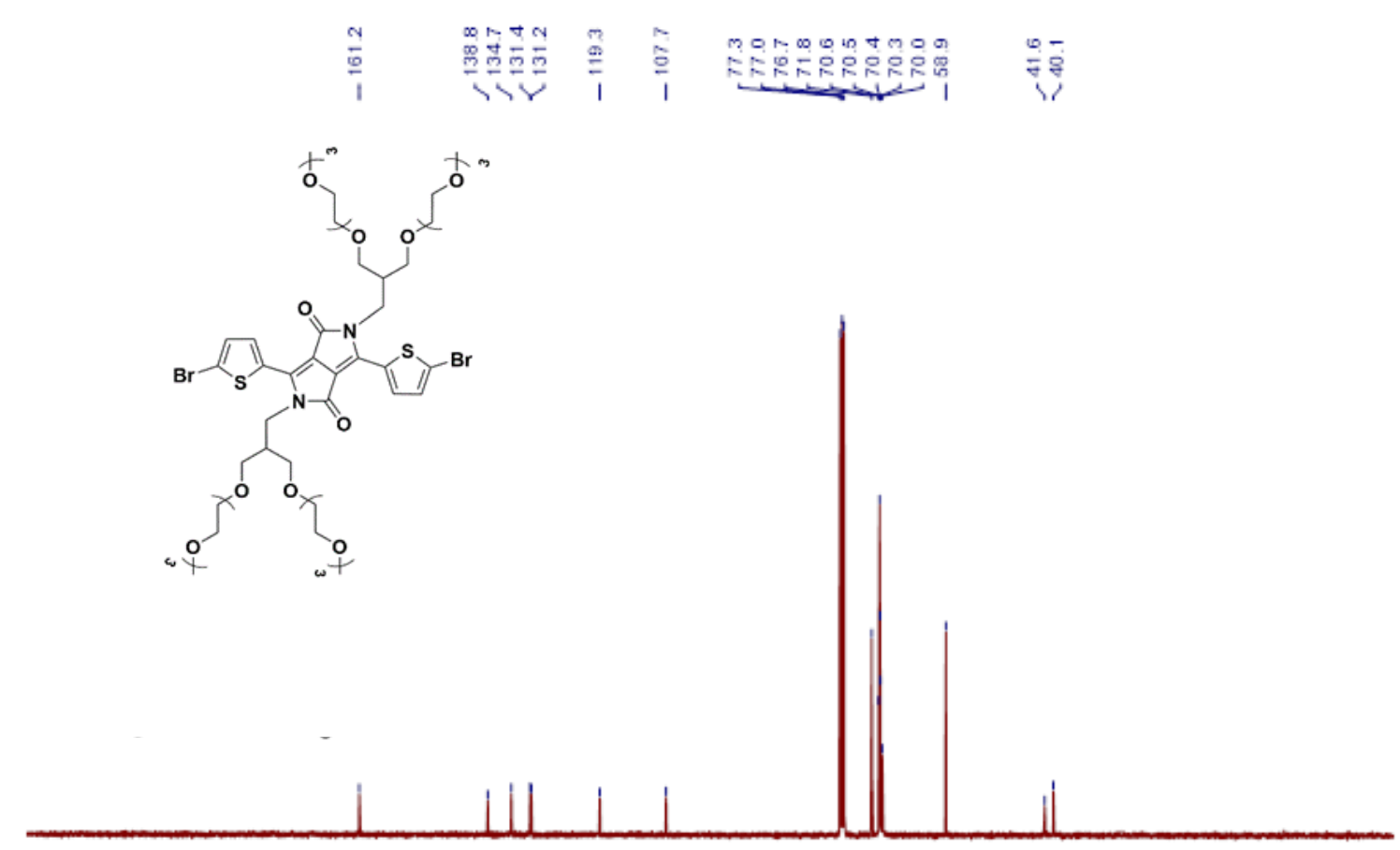

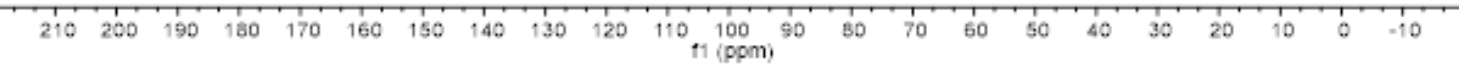

Figure S21 ${ }^{13} \mathrm{C}$ NMR spectrum of $\mathbf{3}$. 


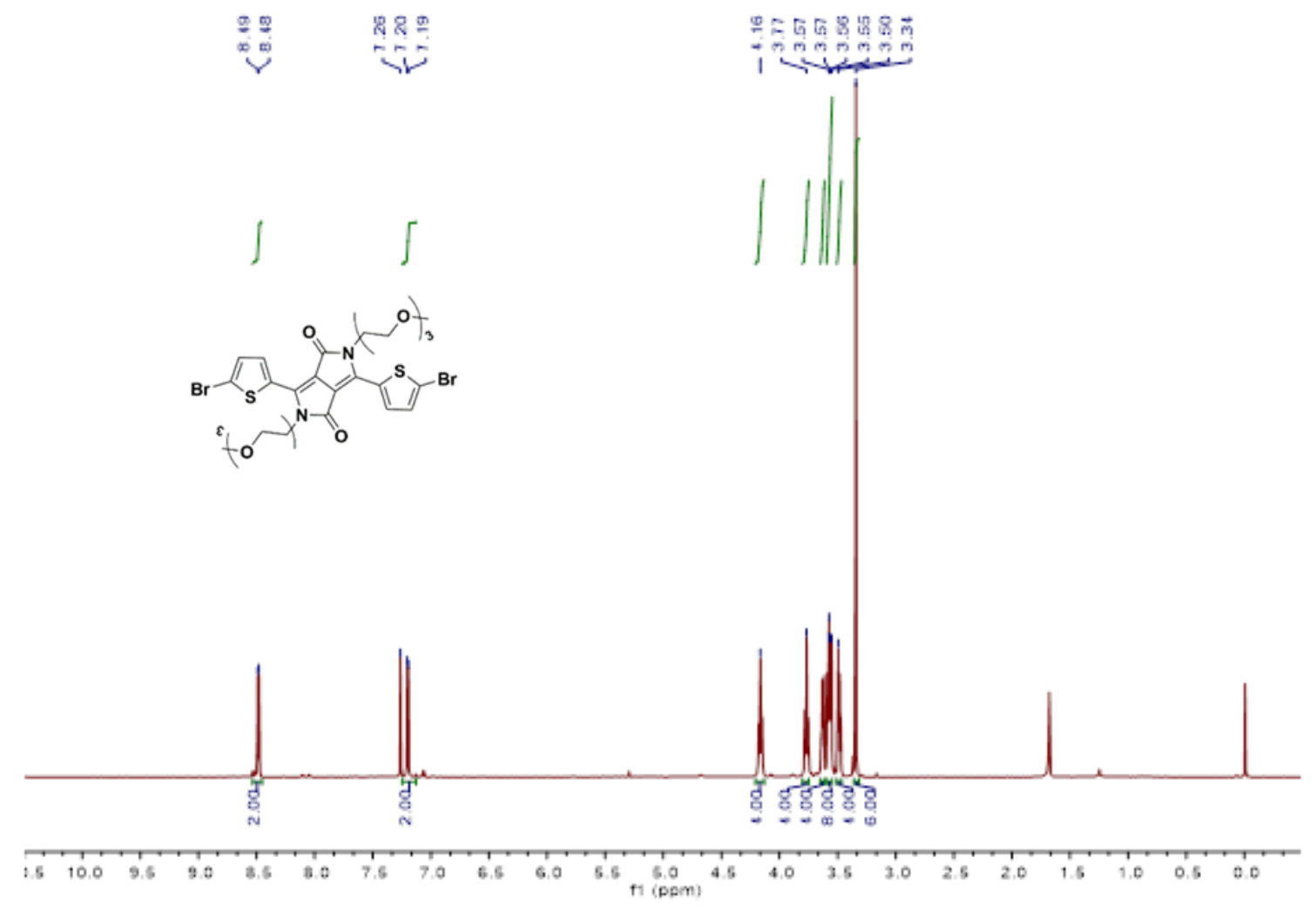

Figure S22 ${ }^{1} \mathrm{H}$ NMR spectrum of 4 .

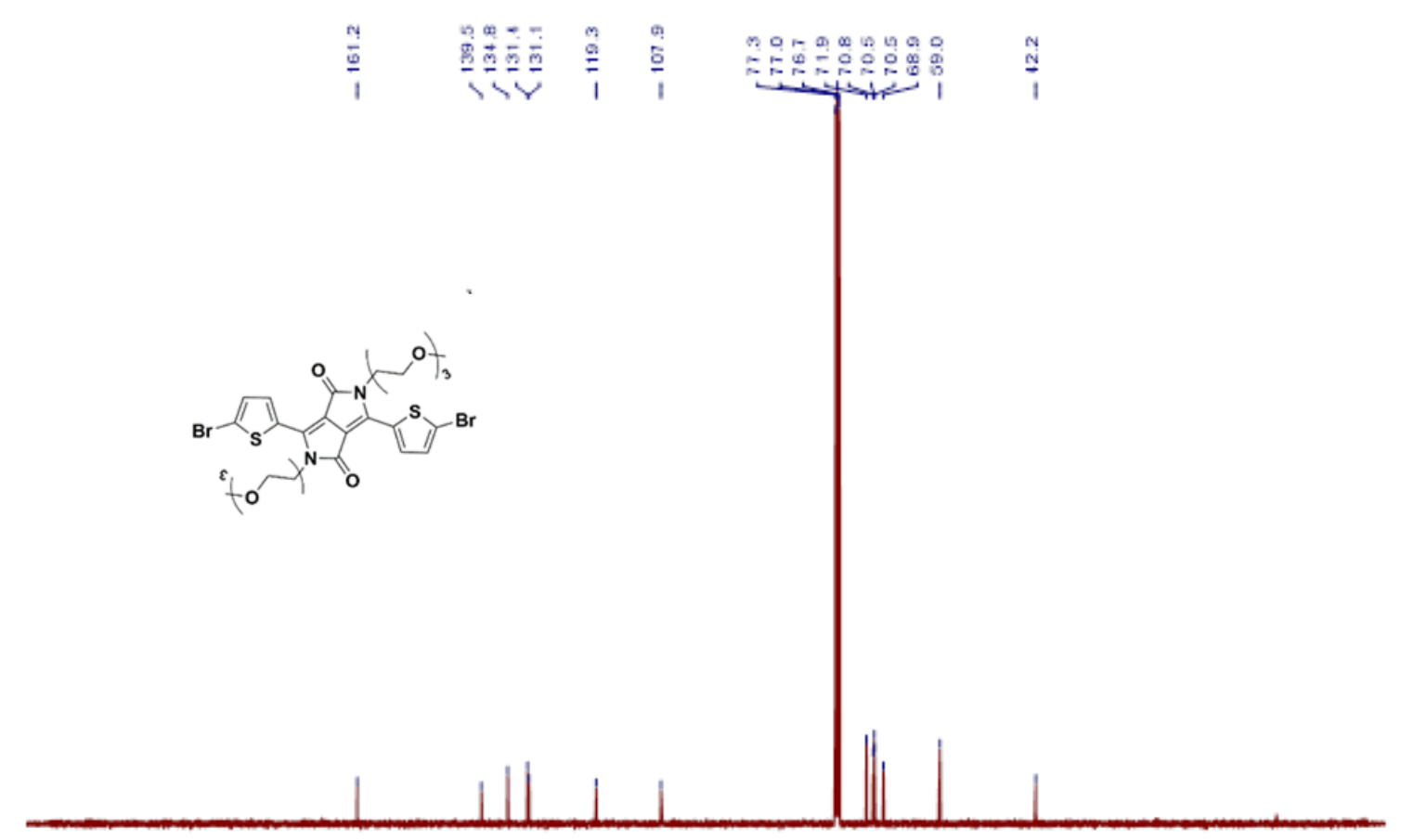

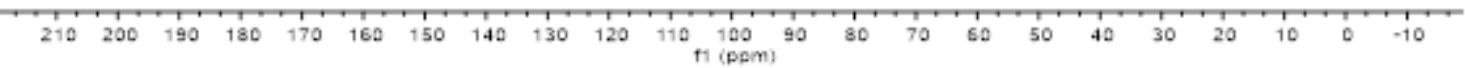

Figure S23 ${ }^{13} \mathrm{C}$ NMR spectrum of 4. 


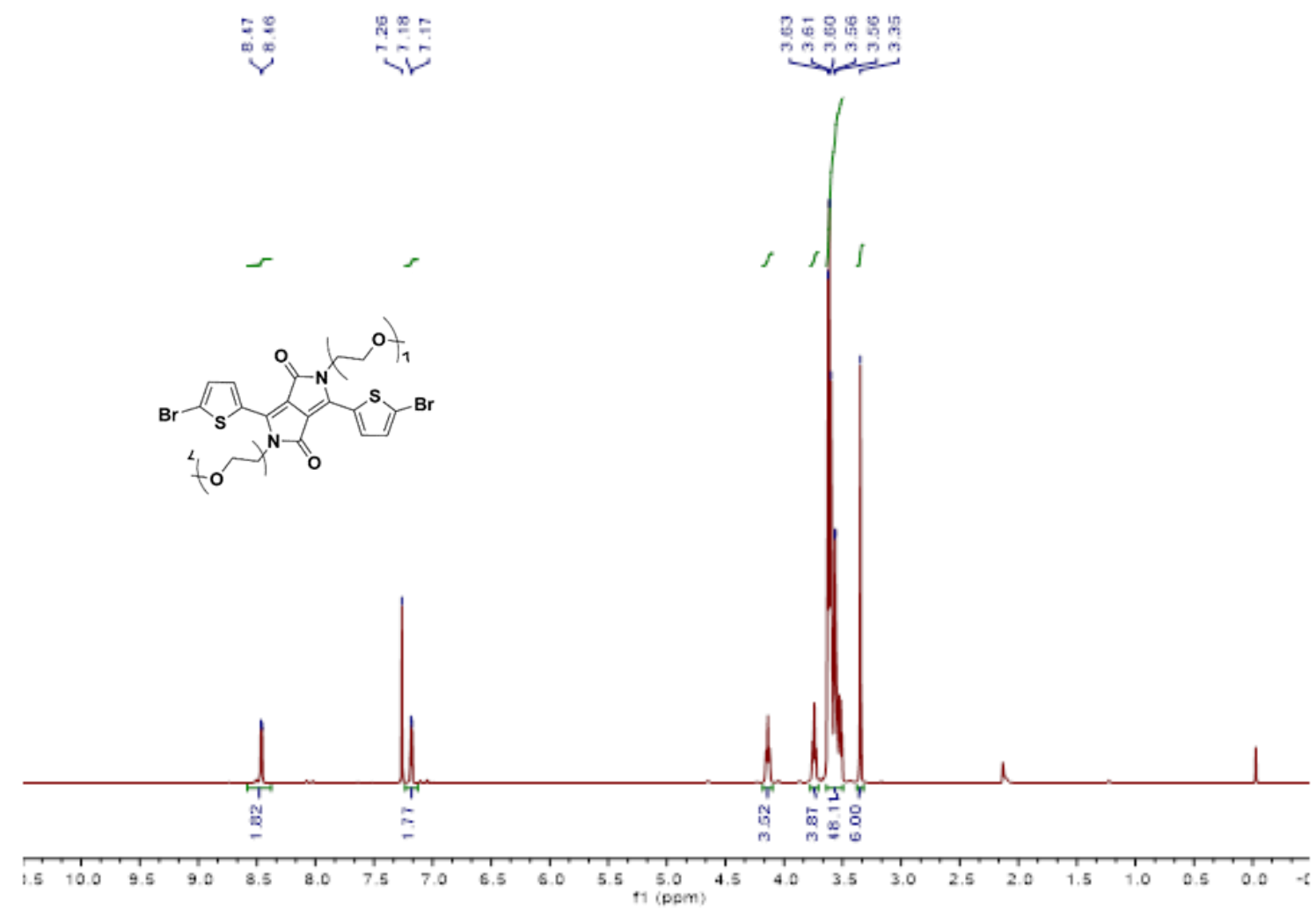

Figure S24 ${ }^{1} \mathrm{H}$ NMR spectrum of 5.

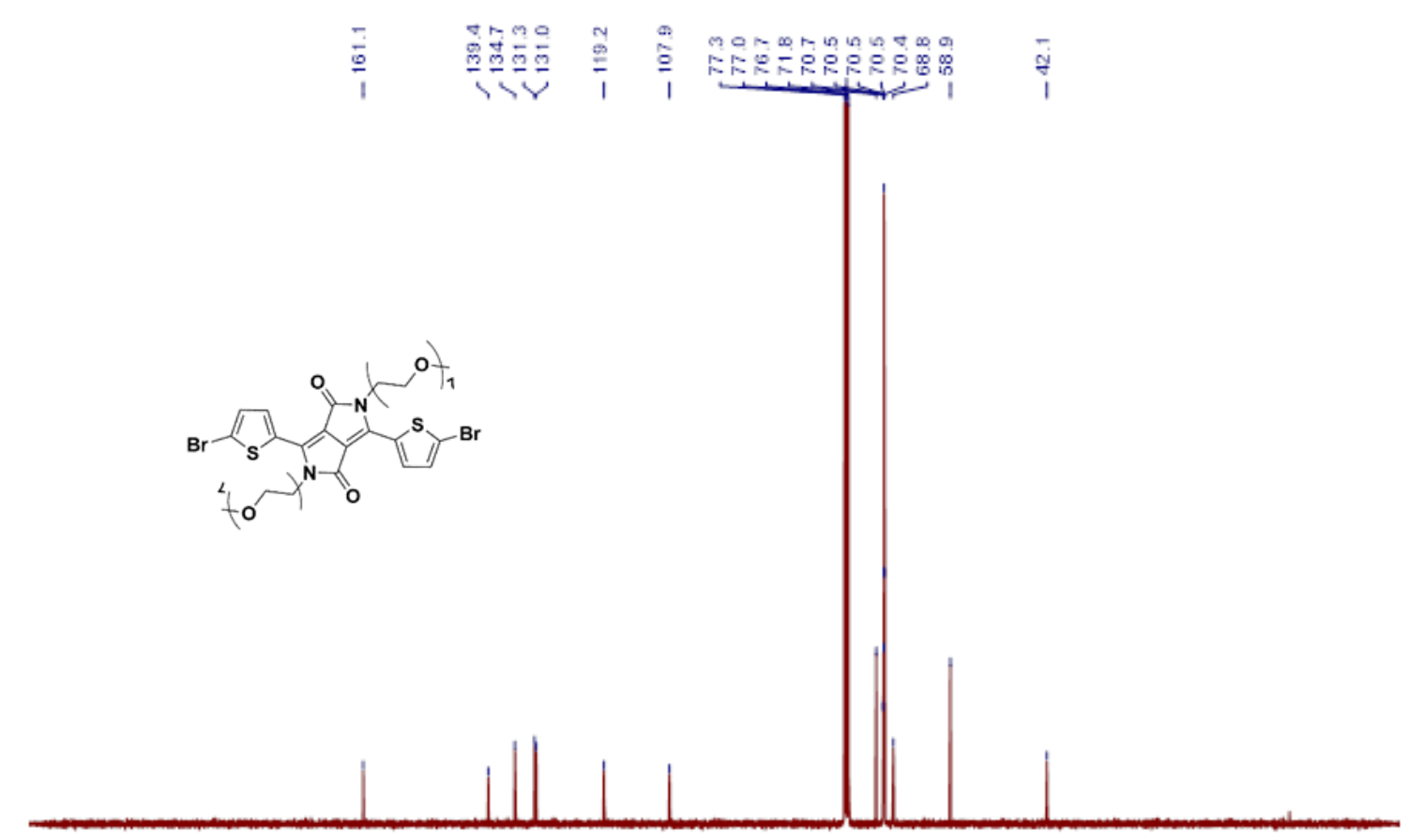

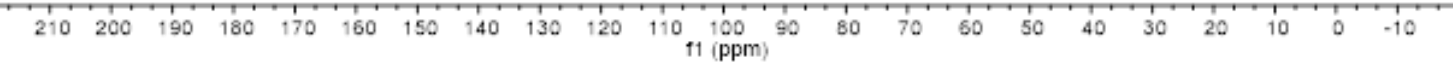

Figure S25 ${ }^{13} \mathrm{C}$ NMR spectrum of 5 . 


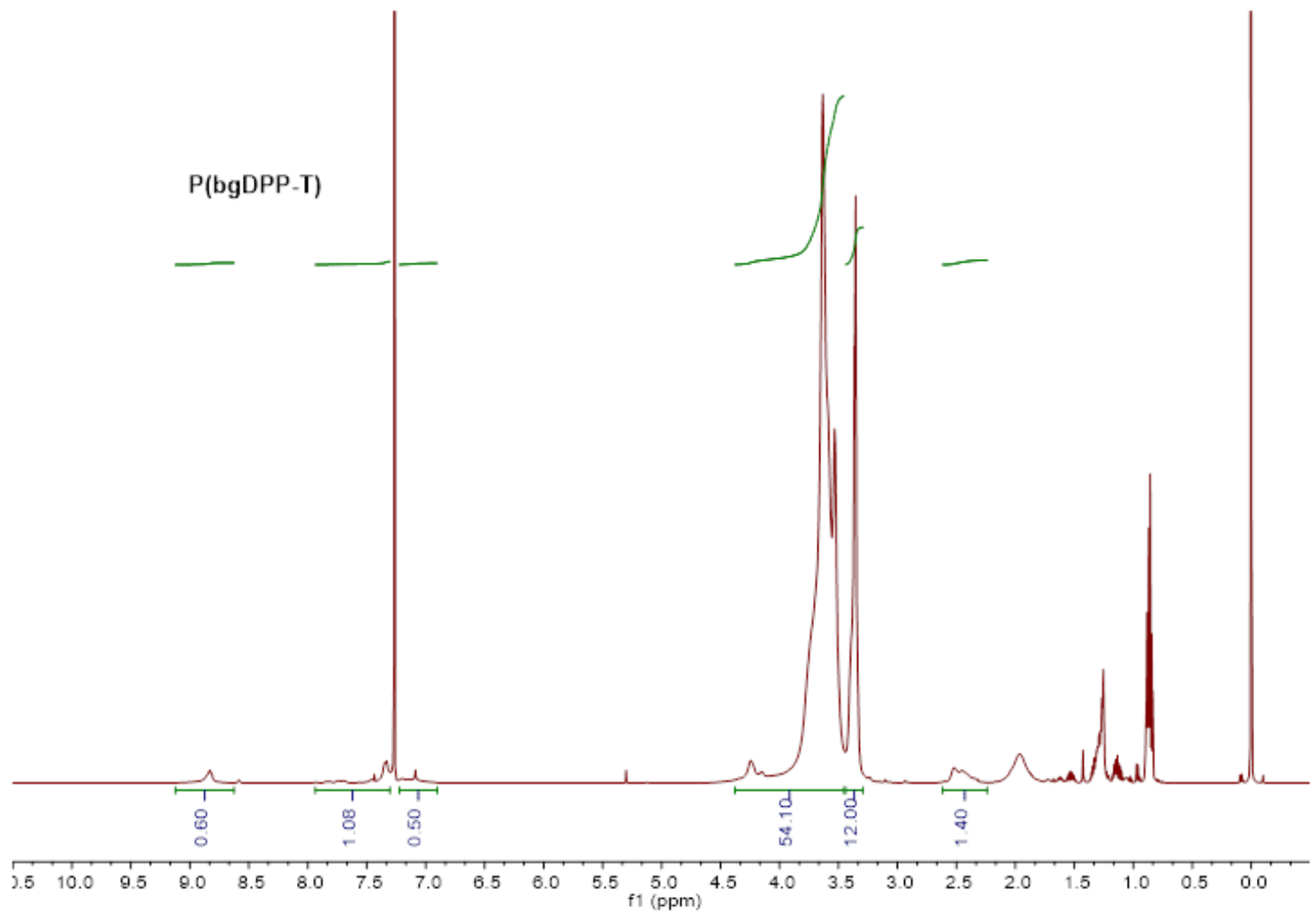

Figure S26 ${ }^{1} \mathrm{H}$ NMR spectrum of $\mathbf{P}(\mathbf{b g D P P}-\mathbf{T})$.

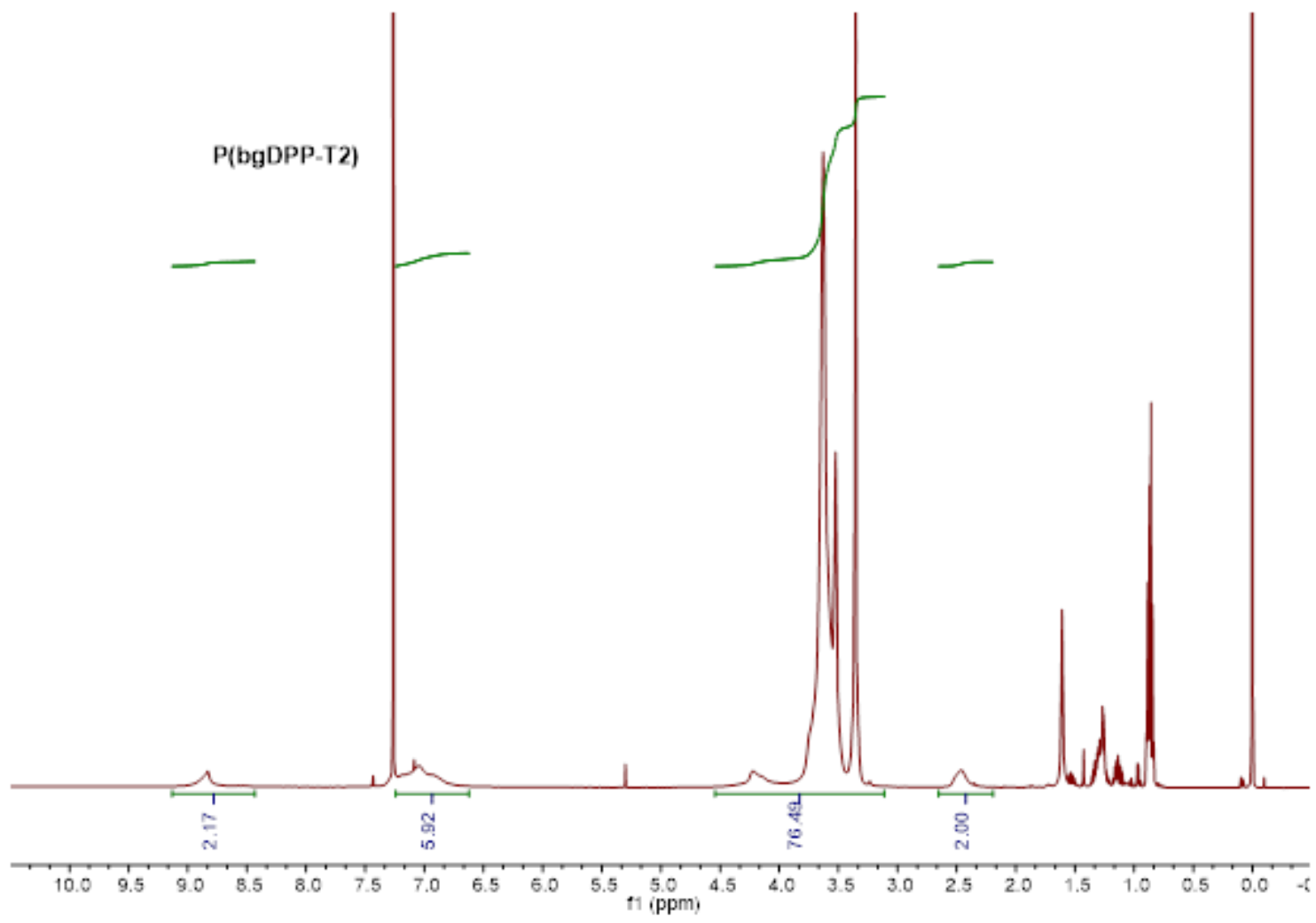

Figure S27 ${ }^{1} \mathrm{H}$ NMR spectrum of P(bgDPP-T2).

S24 


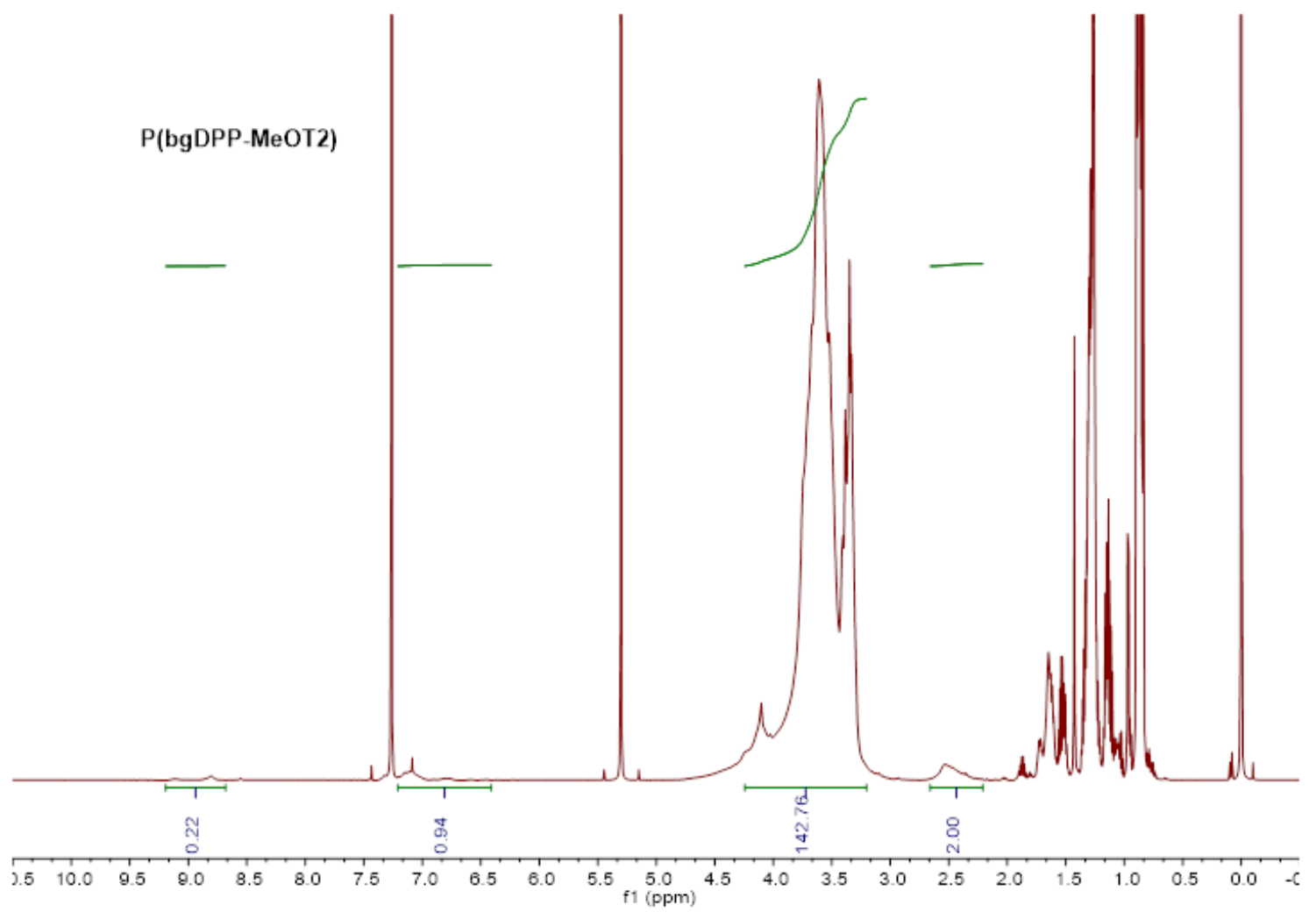

Figure S28 ${ }^{1} \mathrm{H}$ NMR spectrum of P(bgDPP-MeOT2).

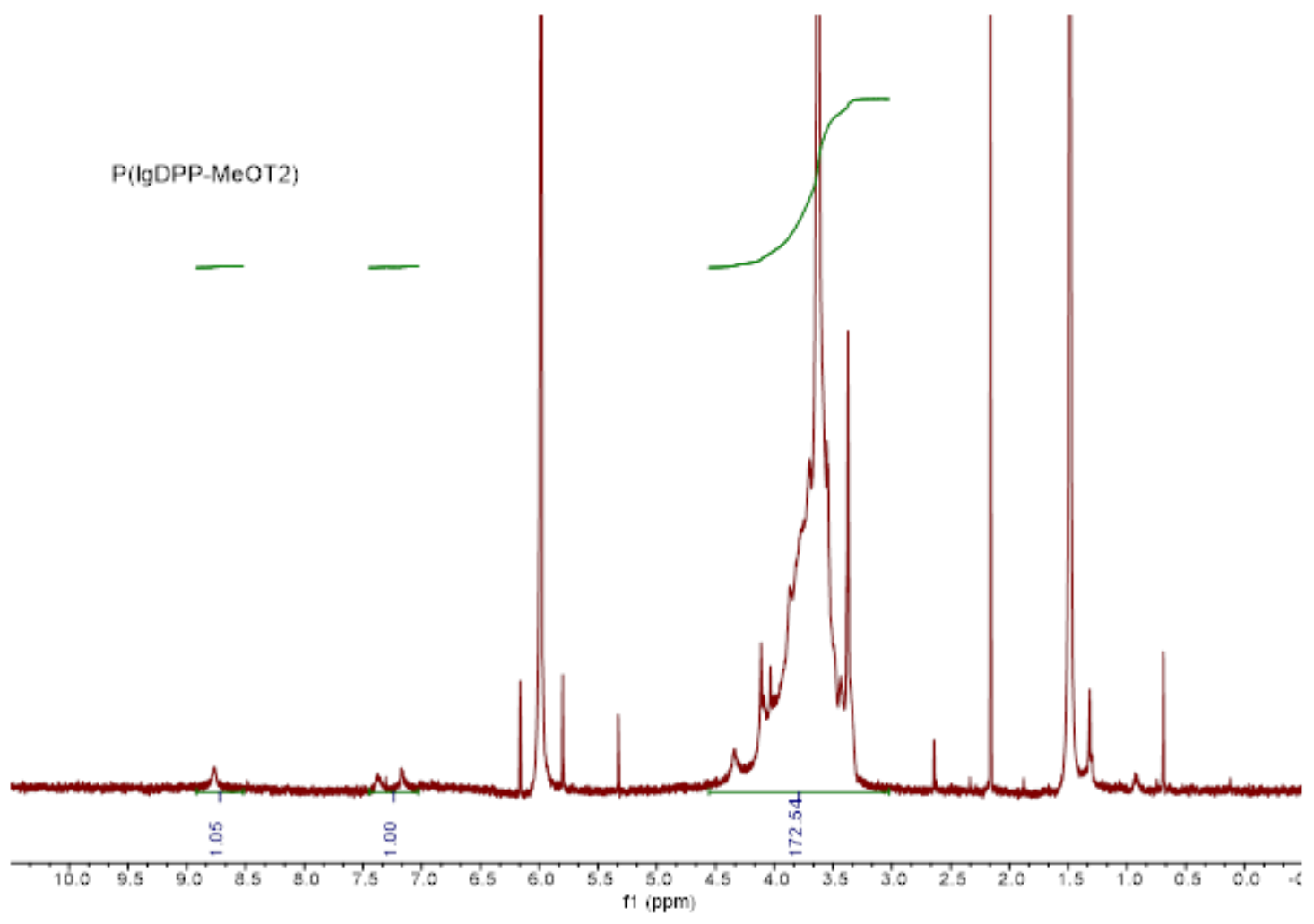

Figure S29 ${ }^{1} \mathrm{H}$ NMR spectrum of P(IgDPP-MeOT2).

S25 


\section{References:}

1. Nielsen, C. B.; Giovannitti, A.; Sbircea, D. T.; Bandiello, E.; Niazi, M. R.; Hanifi, D. A.; Sessolo, M.; Amassian, A.; Malliaras, G. G.; Rivnay, J.; McCulloch, I., Molecular Design of Semiconducting Polymers for High-Performance Organic Electrochemical Transistors. J. Am. Chem. Soc. 2016, 138, 10252-10259.

2. Moser, M.; Savagian, L. R.; Savva, A.; Matta, M.; Ponder, J. F.; Hidalgo, T. C.; Ohayon, D.; Hallani, R.; Reisjalali, M.; Troisi, A.; Wadsworth, A.; Reynolds, J. R.; Inal, S.; McCulloch, I., Ethylene Glycol-Based Side Chain Length Engineering in Polythiophenes and its Impact on Organic Electrochemical Transistor Performance. Chem. Mater. 2020, 32, 6618-6628.

3. Giovannitti, A.; Sbircea, D. T.; Inal, S.; Nielsen, C. B.; Bandiello, E.; Hanifi, D. A.; Sessolo, M.; Malliaras, G. G.; McCulloch, I.; Rivnay, J., Controlling the mode of operation of organic transistors through side-chain engineering. Proc. Natl. Acad. Sci. U. S. A. 2016, 113, 12017-12022.

4. Savva, A.; Hallani, R.; Cendra, C.; Surgailis, J.; Hidalgo, T. C.; Wustoni, S.; Sheelamanthula, R.; Chen, X.; Kirkus, M.; Giovannitti, A.; Salleo, A.; McCulloch, I.; Inal, S., Balancing Ionic and Electronic Conduction for High - Performance Organic Electrochemical Transistors. Adv. Funct. Mater. 2020, 30, 1907657.

5. Khodagholy, D.; Rivnay, J.; Sessolo, M.; Gurfinkel, M.; Leleux, P.; Jimison, L. H.; Stavrinidou, E.; Herve, T.; Sanaur, S.; Owens, R. M.; Malliaras, G. G., High transconductance organic electrochemical transistors. Nat. Commun. 2013, 4, 2133.

6. Inal, S.; Rivnay, J.; Leleux, P.; Ferro, M.; Ramuz, M.; Brendel, J. C.; Schmidt, M. M.; Thelakkat, M.; Malliaras, G. G., A high transconductance accumulation mode electrochemical transistor. Adv. Mater. 2014, 26, 7450-7455.

7. Inal, S.; Malliaras, G. G.; Rivnay, J., Benchmarking organic mixed conductors for transistors. Nat. Commun. 2017, 8, 1767.

8. Wang, Y.; Zeglio, E.; Liao, H.; Xu, J.; Liu, F.; Li, Z.; Maria, I. P.; Mawad, D.; Herland, A.; McCulloch, I.; Yue, W., Hybrid Alkyl-Ethylene Glycol Side Chains Enhance Substrate Adhesion and Operational Stability in Accumulation Mode Organic Electrochemical Transistors. Chem. Mater. 2019, 31, 9797-9806.

9. Sun, H.; Vagin, M.; Wang, S.; Crispin, X.; Forchheimer, R.; Berggren, M.; Fabiano, S., Complementary Logic Circuits Based on High-Performance n-Type Organic Electrochemical Transistors. Adv. Mater. 2018, 30, 1704916.

10. Lill, A. T.; Cao, D. X.; Schrock, M.; Vollbrecht, J.; Huang, J.; Nguyen-Dang, T.; Brus, V. V.; Yurash, B.; Leifert, D.; Bazan, G. C.; Nguyen, T. Q., Organic Electrochemical Transistors Based on the Conjugated Polyelectrolyte PCPDTBT-SO3 K (CPE-K). Adv. Mater. 2020, DOI:10.1002/adma.201908120.

11. Giovannitti, A.; Rashid, R. B.; Thiburce, Q.; Paulsen, B. D.; Cendra, C.; Thorley, K.; Moia, D.; Mefford, J. T.; Hanifi, D.; Weiyuan, D.; Moser, M.; Salleo, A.; Nelson, J.; McCulloch, I.; Rivnay, J., Energetic Control of Redox-Active Polymers toward Safe Organic Bioelectronic Materials. Adv. Mater. 2020, 32, e1908047.

12. Buendia, J.; Sanchez, L., Solvent-dependent disassembly of amphiphilic OPE-based tricarboxamides.

Org. Lett. 2013, 15, 5746-5749. 
